\title{
FRONT SOLUTIONS FOR BISTABLE DIFFERENTIAL-DIFFERENCE EQUATIONS WITH INHOMOGENEOUS DIFFUSION*
}

\author{
A. R. HUMPHRIES ${ }^{\dagger}$, BRIAN E. MOORE $^{\ddagger}$, AND ERIK S. VAN VLECK ${ }^{\S}$
}

\begin{abstract}
We consider a bistable differential-difference equation with inhomogeneous diffusion. Employing a piecewise linear nonlinearity, often referred to as McKean's caricature of the cubic, we construct front solutions which correspond, in the case of homogeneous diffusion, to monotone traveling front solutions or, in the case of propagation failure, to stationary front solutions. A general form for these fronts is given for essentially arbitrary inhomogeneous discrete diffusion, and conditions are given for the existence of solutions to the original discrete Nagumo equation. The specific case of one defect is considered in depth, giving a complete understanding of propagation failure and a grasp on changes in wave speed. Insight into the dynamic behavior of these front solutions as a function of the magnitude and relative position of the defects is obtained with the assistance of numerical results.
\end{abstract}

Key words. traveling fronts, propagation failure, inhomogeneities, bistable equation

AMS subject classifications. 35K57, 73D99

DOI. $10.1137 / 100807156$

1. Introduction. Bistable differential-difference equations (also referred to as spatially discrete reaction-diffusion equations in the literature) occur naturally in many fields of study, including biology, physiology, and material science, in which there is an inherent spatial length scale. Mathematically, it is typically assumed that the problem is spatially homogeneous so that the resulting spatially discrete equations are homogeneous in space. It is then natural to consider traveling front solutions in which the solution at a given time is a translate of itself at any other time.

Our aim is to consider the case of inhomogeneous discrete diffusion which could occur due to small or large differences in the diffusive properties of the media, partly motivated by modeling myelinated axons [3]. Throughout the article, we refer to these localized changes in homogeneous diffusion as defects. To achieve our aim, we use a specific bistable nonlinearity which, after derivation of possible solutions, allows for a study of the behavior of these front solutions that correspond to traveling front solutions in the case of homogeneous diffusion. In particular, we study the behavior of front solutions near the defect and characterize defects that cause propagation failure and/or a decrease in propagation speed.

Our approach takes advantage of the fact that, under certain conditions with the piecewise linear bistable nonlinearity (McKean's caricature of the cubic), the nonlinear problem reduces to a linear inhomogeneous problem. This problem can then be solved using Jacobi operator theory for the steady-state equation, or transform

${ }^{*}$ Received by the editors September 1, 2010; accepted for publication (in revised form) April 14, 2011; published electronically August 9, 2011. This work was supported in part under NSF grants DMS-0139824, DMS-0513438, and DMS-0812800 and by NSERC grant 204543.

http://www.siam.org/journals/siap/71-4/80715.html

${ }^{\dagger}$ Department of Mathematics and Statistics, McGill University, Montreal, QC H3A 2K6, Canada (Tony.Humphries@mcgill.ca).

${ }^{\ddagger}$ Department of Mathematics, University of Central Florida, Orlando, FL 32816 (bmoore@math. ucf.edu).

$\S$ Department of Mathematics, University of Kansas, Lawrence, KS 66045 (evanvleck@math.ku. edu). 
techniques for the time dependent equation. We show that this approach can still be employed in the case of inhomogeneous discrete diffusion, with some important modifications. In particular, since we no longer have translational invariance in the case of inhomogeneous diffusion, we must find the family of solutions that arise as the front moves through the medium. This is made possible through the choice of nonlinearity and by the solution profile that we seek.

Besides the diffusion coefficients, there are two natural parameters to consider, the detuning parameter $a$, that controls the bistable nonlinearity, and the wave speed $c$. Fronts that are far from defects have constant wave speeds, but as fronts approach defects, the speed and shape of the front change. Hence, we allow solutions with wave speeds that depend on both space and time. By considering a specific bistable nonlinearity where explicit solutions may be obtained, a general framework is laid out that should prove useful in extending our results to more general cases of inhomogeneous discrete diffusion.

An alternative to discrete diffusion is to consider a continuous problem with inhomogeneous diffusion (see the work of Scheel and Van Vleck [28]) or an inhomogeneous reaction term (see the work of Keener [20, 21]). In this case, the analogue of homogeneous discrete diffusion is a spatially periodic continuous inhomogeneous term. The present paper was motivated in part by the work of Lewis and Keener [22] in which they studied wave-block due to depressed excitability in a single region using inhomogeneous reaction and diffusion terms with a geometric technique. Extensions of these results may be found in the work of Yang et al. [36], where the cases of two and three regions of depressed excitability are considered, and the work of Aronson, Mantzaris, and Othmer [1], which considers media with an arbitrary number of depressed regions. Other approaches to inhomogeneous diffusion/reaction in continuous media include the work of Fife and Peletier [16], Pauwelussen [26], and Sneyd and Sherratt [31].

There is a large body of literature on existence, uniqueness, and stability of traveling fronts for spatially discrete reaction-diffusion equations. Notable is the work of Bates, Chen, and Chmaj [2] and Chow, Mallet-Paret, and Shen [8] for bistable Nagumo-type differential-difference equations. A general theory for existence of solutions to mixed-type differential equations that occur in equations with both backward and forward delays has been developed by Mallet-Paret [23, 24]. Important original work on traveling front solutions to discrete bistable equations with homogeneous diffusion includes that of Keener [19] and Zinner [37, 38] and the work of Cahn [4] for diffuse interfaces. Regarding other work on homogeneous discrete media, Shen [29, 30] considers time dependent reaction terms, Carpio and Bonilla [6] consider waves near the boundary for propagation failure, and Chen, Guo, and $\mathrm{Wu}[7]$ have developed a general theory for bistable dynamics in periodic media.

The use of McKean's caricature of the cubic for continuous space problems dates back to the work of McKean [25] and Rinzel and Keller [27] and the subsequent work of Wang [34, 35] and Feroe [15]. More recent work in the case of discrete diffusion includes that in [5] and [14]. Its use has proved advantageous in a variety of different contexts including neuronal networks [9] and martensitic phase transitions [33].

The paper is outlined as follows. In section 2, we present background and basics behind our approach. Section 3 contains the derivation of solutions for the steadystate problem, which implies necessary and sufficient conditions for fronts to fail to propagate and describes changes in the interval of propagation failure in the presence of inhomogeneous diffusion. In section 4 we derive candidate solutions with nonzero wave speed using transform techniques, providing a description of changes in the wave 
speed as the front moves through the defect region. We summarize our results and state conclusions in section 5 .

2. Preliminaries. Following [12], we consider traveling front solutions for a bistable differential-difference equation with diffusion coefficients that vary on an integer lattice,

$$
\dot{u}_{j}(t)=L u_{j}(t)-f\left(u_{j}(t)\right),
$$

where $u_{j}(t)$ maps $\mathbb{R}^{+} \cup\{0\} \rightarrow \mathbb{R}, j \in \mathbb{Z}$ indicates a particular element of the onedimensional lattice, and $\dot{u}$ denotes differentiation of $u$ with respect to $t$. We represent variable diffusion over the spatially discrete domain with the operator $L$, a difference Laplacian operator of the form

$$
L u_{j}(t)=\alpha_{j}\left[u_{j+1}(t)-u_{j}(t)\right]+\alpha_{j-1}\left[u_{j-1}(t)-u_{j}(t)\right],
$$

where $\alpha_{j} \in \mathbb{R}^{+}, j \in \mathbb{Z}$. The nonlinearity $f: \mathbb{R} \rightarrow \mathbb{R}$ is typically taken to be the derivative of a double-well potential, such as the cubic

$$
f(u)=u(u-a)(u-1),
$$

or the McKean caricature of the cubic [25, 27], which is the piecewise linear function

$$
f(u)=u-h(u-a),
$$

where $h$ is the Heaviside function

$$
h(u)= \begin{cases}0, & u<0 \\ {[0,1],} & u=0 \\ 1, & u>0\end{cases}
$$

considered to be set-valued when $u=0$, and $a \in(0,1)$ is known as the detuning parameter.

We define the diffusion coefficients to be

$$
\alpha_{j}= \begin{cases}\alpha_{j}, & -\mathrm{m} \leqslant j \leqslant \mathrm{n}, \\ \alpha, & j<-\mathrm{m} \quad \text { or } \quad j>\mathrm{n},\end{cases}
$$

for $\mathrm{m}, \mathrm{n} \in\{0\} \cup \mathbb{Z}^{+}$. For the case of inhomogeneous diffusion, in which one or more of the $\alpha_{j} \neq \alpha$, the front may change form as it moves through the medium. Thus, in contrast with the case of homogeneous discrete diffusion, we do not have translation invariance and must consider fronts at different points in the medium. If the detuning parameter $a$ is kept fixed, then with inhomogeneous media one expects that both the wave form and the wave speed will depend on the position of the front in the medium. In other words, we expect the wave speed to depend on both time and position in the lattice.

As a result, we make the traveling wave ansatz

$$
u_{j}(t)=\varphi\left(\xi_{j} ; \xi^{*}\right), \quad \xi_{j}=j-c_{j}(t) .
$$

In the case of homogeneous diffusion, we have $c_{j}(t)=c t$ with a constant wave speed $c$, and $t \in \mathbb{R}$ implies $\xi_{j} \in \mathbb{R}$. Thus, $\varphi: \mathbb{R} \rightarrow[0,1]$, and the same is true in the case of 
inhomogeneous diffusion, provided $c_{j}(t)$ is continuous in $t$. The parameter $\xi^{*}$ denotes the position of the front in the medium. We choose a particular wave form by setting

$$
a:=\varphi\left(\xi^{*} ; \xi^{*}\right),
$$

so that $\xi^{*}$ is the spatial location at which $\varphi$ takes the value $a$, and we seek solutions that satisfy

$$
\varphi\left(x ; \xi^{*}\right)<a \text { for } x<\xi^{*} \quad \text { and } \quad \varphi\left(x ; \xi^{*}\right)>a \text { for } x>\xi^{*} .
$$

Since we use the piecewise linear function (2.4)-(2.5), $x=\xi^{*}$ is the point at which the Heaviside function is activated, and the nonlinearity (2.4) may be written as a linear inhomogeneous term

$$
f\left(\varphi\left(x ; \xi^{*}\right)\right)=\varphi\left(x ; \xi^{*}\right)-h\left(x-\xi^{*}\right),
$$

which is independent of $a$. With a bistable nonlinearity, it is natural to impose the boundary conditions

$$
\lim _{x \rightarrow-\infty} \varphi\left(x ; \xi^{*}\right)=0, \quad \quad \lim _{x \rightarrow \infty} \varphi\left(x ; \xi^{*}\right)=1 .
$$

We consider the solutions in two different cases, which are

$$
\begin{array}{ll}
c_{j}(t) \neq 0 & \forall j \in \mathbb{Z}, \\
c_{j}(t)=0 & \forall j \in \mathbb{Z} .
\end{array}
$$

We begin with zero wave speed, (2.13). The case of nonzero wave speed, (2.12), is treated in section 4 .

3. Derivation of solutions for zero wave speed. Consider solutions to (2.1) with nonlinearity (2.10) in the case of (2.13). In this case, finding solutions is equivalent to finding the steady states of (2.1), which means solving the difference equation

$$
\alpha_{k}\left(\phi_{k+1}-\phi_{k}\right)+\alpha_{k-1}\left(\phi_{k-1}-\phi_{k}\right)-\phi_{k}=-h_{k} \quad \forall k \in \mathbb{Z},
$$

with boundary conditions

$$
\lim _{k \rightarrow-\infty} \phi_{k}=0 \quad \text { and } \quad \lim _{k \rightarrow \infty} \phi_{k}=1,
$$

where $h_{k}=h\left(k-\xi^{*}\right)$. Equation (3.1) is an infinite-dimensional tridiagonal linear system, and general solutions may be derived using Jacobi operator theory. We also mention that solutions to (3.1) are also stationary front solutions for spatially discrete wave equations $\ddot{u}_{j}(t)=L u_{j}(t)-f\left(u_{j}(t)\right)$, and spatially discrete Schrödinger equations $i \dot{u}_{j}(t)=L u_{j}(t)-f\left(u_{j}(t)\right)$, where $f\left(u_{j}(t)\right)$ is a McKean nonlinearity.

We have dropped the $\xi^{*}$-dependence in (3.1) but remind the reader of the importance of this parameter, as it allows us to consider stationary fronts at different positions in the media. Let $k^{*}$ be the integer satisfying $k^{*}=\left\lfloor\xi^{*}\right\rfloor$. Solutions to (3.1) depend on whether the interface lies on a lattice point or between lattice points, which respectively correspond to the following cases according to $(2.8)-(2.9)$ :

$$
\begin{aligned}
& \xi^{*} \in \mathbb{Z} \quad \Longleftrightarrow \quad k^{*}=\xi^{*} \quad \text { implies } \quad a=\phi_{k^{*}} \quad \text { with } h_{k^{*}}=[0,1] . \\
& \xi^{*} \notin \mathbb{Z} \quad \Longleftrightarrow \quad k^{*}<\xi^{*}<k^{*}+1 \quad \text { implies } a \in\left(\phi_{k^{*}}, \phi_{k^{*}+1}\right) \text { with } h_{k^{*}}=0 .
\end{aligned}
$$


In the case $\alpha_{k}=\alpha$ for all $k$, there are only two steady-state solutions of (2.1), one unstable corresponding to (3.3) and one stable corresponding to (3.4), and our choice of $\xi^{*}$ is important only for determining which of the two we are considering (see [11]). This is a result of the translational invariance for solutions of the homogeneous diffusion equation. In the case of inhomogeneous diffusion, our choice of $\xi^{*}$ determines not only whether or not the interface falls on a lattice point, but also the position of the front relative to the defect region. This gives important information as to where fronts may become pinned due to inhomogeneities in the medium.

3.1. Homogeneous diffusion. Derivation of general solutions for (3.1) is based on general solutions for the problem with homogeneous diffusion. Hence, we begin by presenting a general approach for finding solutions for $\alpha_{k}=\alpha>0$ for all $k \in \mathbb{Z}$. Solutions for this case have been discussed in [5] as a limiting case of the nonzero wave speed solutions, and also by Elmer [10], who considered stationary fronts of this equation with different nonlinearities.

We seek a general solution to

$$
-\alpha \phi_{k+1}+(1+2 \alpha) \phi_{k}-\alpha \phi_{k-1}=h_{k} \quad \forall k \in \mathbb{Z},
$$

which can be written in terms of the general solution to the problem with zero righthand side,

$$
-\alpha \phi_{k+1}+(1+2 \alpha) \phi_{k}-\alpha \phi_{k-1}=0 \quad \forall k \in \mathbb{Z}
$$

and a particular solution of (3.5). This is done following the results of Teschl [32]. Start by considering

$$
\phi_{k+1}+\phi_{k-1}=2 \mu \phi_{k}
$$

which is just (3.6) for

$$
\mu=\frac{1}{2 \alpha}(1+2 \alpha)
$$

Since (3.7) is a second order difference equation, the space of solutions is two-dimensional, and any solution can be written in the form

$$
\phi_{k}=\phi_{k_{0}} \rho\left(k-k_{0}\right)+\phi_{k_{0}+1} \sigma\left(k-k_{0}\right)
$$

for any starting point $k_{0}$, where the functions $\rho\left(k-k_{0}\right)$ and $\sigma\left(k-k_{0}\right)$ are a set of linearly independent solutions of (3.7), known as the fundamental solutions, which satisfy the initial conditions

$$
\rho(0)=1, \quad \rho(1)=0, \quad \sigma(0)=0, \quad \sigma(1)=1 .
$$

In order to find expressions for $\rho\left(k-k_{0}\right)$ and $\sigma\left(k-k_{0}\right)$ substitute $\phi_{k}=\lambda^{k}$ into (3.7) to get $\lambda^{2}-2 \mu \lambda+1=0$, which implies $\lambda_{ \pm}=\mu \pm \sqrt{\mu^{2}-1}$. Noting that $\lambda_{+} \lambda_{-}=1$, we set

$$
\lambda=\mu+\sqrt{\mu^{2}-1}=1+\frac{1}{2 \alpha}+\frac{1}{2 \alpha} \sqrt{1+4 \alpha},
$$

so the roots are $\lambda$ and $\lambda^{-1}$ with $\lambda>1>\lambda^{-1}$. Using the initial conditions (3.10), this implies that the fundamental solutions are given by

$$
\rho\left(k-k_{0}\right)=\frac{\lambda^{k_{0}+1-k}-\lambda^{k-k_{0}-1}}{\lambda-\lambda^{-1}} \quad \text { and } \quad \sigma\left(k-k_{0}\right)=\frac{\lambda^{k-k_{0}}-\lambda^{k_{0}-k}}{\lambda-\lambda^{-1}},
$$


and the general solution of (3.6) is obtained by substituting these quantities into (3.9). Without loss of generality we set $k_{0}=k^{*}$, and following [32], the general solution of (3.5) takes the form

$$
\phi_{k}=\phi_{k^{*}} \rho\left(k-k^{*}\right)+\phi_{k^{*}+1} \sigma\left(k-k^{*}\right)+ \begin{cases}\frac{-1}{\alpha} \sum_{j=k^{*}+1}^{k} h_{j} \sigma(k-j), & k>k^{*} \\ 0, & k=k^{*} \\ \frac{1}{\alpha} \sum_{j=k+1}^{k^{*}} h_{j} \sigma(k-j), & k<k^{*}\end{cases}
$$

where it is a simple calculation to check that

$$
\phi_{k}= \begin{cases}\frac{-1}{\alpha} \sum_{j=k^{*}+1}^{k} h_{j} \sigma(k-j), & k>k^{*} \\ 0, & k=k^{*} \\ \frac{1}{\alpha} \sum_{j=k+1}^{k^{*}} h_{j} \sigma(k-j), & k<k^{*}\end{cases}
$$

is a particular solution of (3.5). This formulation of the solution will become important in the case of inhomogeneous diffusion.

To simplify (3.13), notice that the discrete Heaviside function takes the form

$$
h_{k}=\left\{\begin{array}{ll}
1, & k>k^{*}, \\
h_{k^{*}}, & k=k^{*}, \\
0, & k<k^{*},
\end{array} \quad \text { where } \quad h_{k^{*}}= \begin{cases}{[0,1],} & \xi^{*} \in \mathbb{Z}, \\
0, & \xi^{*} \notin \mathbb{Z} .\end{cases}\right.
$$

Due to translational invariance of solutions, we may consider the two cases (3.3) and (3.4) by taking $k^{*}=0$, without loss of generality. Hence, the solution (3.13) becomes

$$
\phi_{k}=\phi_{0} \rho(k)+\phi_{1} \sigma(k)+ \begin{cases}\frac{-1}{\alpha} \sum_{j=1}^{k} \sigma(k-j), & k>0 \\ 0, & k=0 \\ \frac{1}{\alpha} h_{0} \sigma(k), & k<0\end{cases}
$$

Next we find $\phi_{0}$ and $\phi_{1}$ to obtain an explicit form of (3.15). Using (3.12) for $k<0$, rewrite $(3.15)$ as

$$
\phi_{k}=\frac{\lambda^{-k}}{\lambda-\lambda^{-1}}\left(\lambda \phi_{0}-\phi_{1}-\frac{h_{0}}{\alpha}\right)-\frac{\lambda^{k}}{\lambda-\lambda^{-1}}\left(\lambda^{-1} \phi_{0}-\phi_{1}-\frac{h_{0}}{\alpha}\right) .
$$

Hence, to satisfy the boundary condition (3.2) as $k \rightarrow-\infty$, we require

$$
\lambda \phi_{0}-\phi_{1}-\frac{h_{0}}{\alpha}=0 .
$$

Similarly, for $k>0$, write $(3.15)$ as

$$
\phi_{k}=\frac{\lambda^{-k}}{\lambda-\lambda^{-1}}\left(\lambda \phi_{0}-\phi_{1}+\frac{1}{\alpha} \sum_{j=1}^{k} \lambda^{j}\right)-\frac{\lambda^{k}}{\lambda-\lambda^{-1}}\left(\lambda^{-1} \phi_{0}-\phi_{1}+\frac{1}{\alpha} \sum_{j=1}^{k} \lambda^{-j}\right) .
$$

Using the equality

$$
(\lambda-1)\left(1-\lambda^{-1}\right)=\frac{1}{\alpha},
$$

Copyright $@$ by SIAM. Unauthorized reproduction of this article is prohibited. 
the boundary condition (3.2) is satisfied as $k \rightarrow \infty$ if and only if

$$
0=\lim _{k \rightarrow \infty}\left(\lambda^{-1} \phi_{0}-\phi_{1}+\frac{1}{\alpha} \sum_{j=1}^{k} \lambda^{-j}\right)=\lambda^{-1} \phi_{0}-\phi_{1}+\frac{1}{\alpha(\lambda-1)}
$$

Solving (3.16), (3.18) for $\phi_{0}$ and $\phi_{1}$ and using (3.17), we obtain

$$
\phi_{0}=v_{0}+h_{0}\left(1-\lambda^{-1}\right) w_{0} \quad \text { and } \quad \phi_{1}=w_{0}+h_{0}\left(1-\lambda^{-1}\right) v_{0},
$$

where we define

$$
v_{0}=\frac{1}{\lambda+1} \quad \text { and } \quad w_{0}=\frac{\lambda}{\lambda+1}
$$

which satisfy the symmetry property $v_{0}+w_{0}=1$. Substituting $\phi_{0}$ and $\phi_{1}$ into the general solution (3.15) and simplifying gives

$$
\phi_{k}= \begin{cases}1+\left(\phi_{1}-1\right) \lambda^{1-k}, & k>0 \\ \phi_{0} \lambda^{k}, & k \leqslant 0 .\end{cases}
$$

In the case (3.3) with $\xi^{*}=0$, we have $a=\phi_{0}$, and $\left(\phi_{1}-1\right) \lambda=\phi_{0}-1$ shows that the unstable solution is

$$
\phi_{k}= \begin{cases}1+(a-1) \lambda^{-k}, & k \geqslant 0, \\ a \lambda^{k}, & k \leqslant 0 .\end{cases}
$$

Since $a=\phi_{0} \in v_{0}+h_{0}\left(1-\lambda^{-1}\right) w_{0}$ with $h_{0}=[0,1]$, and $v_{0}+\left(1-\lambda^{-1}\right) w_{0}=w_{0}$, this implies $a \in\left[v_{0}, w_{0}\right]$. In the case (3.4), we have $h_{0}=0$, which implies $\phi_{0}=v_{0}$ and $\phi_{1}=w_{0}$, meaning the stable solution is

$$
\phi_{k}= \begin{cases}1-v_{0} \lambda^{1-k}, & k \geqslant 0 \\ v_{0} \lambda^{k}, & k \leqslant 0\end{cases}
$$

and since $0<\xi^{*}<1$ implies $\phi_{0}<a<\phi_{1}$, we obtain $a \in\left(v_{0}, w_{0}\right)$. Therefore, regardless of where the interface lies, $a \in\left[v_{0}, w_{0}\right]$ is a necessary and sufficient condition for existence of fronts with zero wave speed. On the boundary of this interval there is a saddle-node bifurcation $[10,11]$. The interval $\left[v_{0}, w_{0}\right]$ is known as the interval of propagation failure, defined in the following way.

DEFINITION 3.1. The interval of propagation failure is the range of values for the detuning parameter a, determined by (3.3)-(3.4), which yield solutions (called pinned or stationary fronts) of (3.1).

Essentially, $v_{0}$ and $w_{0}$ provide boundaries. Inside these boundaries there are two stationary fronts, one stable and one unstable. On the boundaries these two fronts collide, giving rise to a traveling front [5] whenever $a \notin\left[v_{0}, w_{0}\right]$. More specifically, we know that $a<v_{0}$ gives a left traveling front, corresponding to negative wave speed, and $a>w_{0}$ gives a right traveling front, corresponding to positive wave speed. In sections 3.2 and 3.3 we study this phenomenon for fronts in inhomogeneous media.

3.2. Inhomogeneous diffusion: One defect. Considering the case of a single defect, we seek solutions of the difference equation (3.1), (2.6) with $\mathrm{m}=\mathrm{n}=0$ so that $\alpha_{j}=\alpha$ for $j \neq 0$ but $\alpha_{0} \neq \alpha$. Since solutions of this equation depend on the location of the front relative to the defect, as well as on whether or not $\xi^{*}$ is an integer, we 
consider the general cases $\xi^{*}=k^{*}$ and $k^{*}<\xi^{*}<k^{*}+1$ for $k^{*} \in \mathbb{Z}$. Changing the value of $\xi^{*}$ (respectively, $k^{*}$ ) allows one to consider stationary fronts at different positions relative to the defect, according to the construction (2.8)-(2.9).

To get the general solution of (3.1) we first find the fundamental solutions of

$$
-\alpha_{k} \phi_{k+1}+\left(1+\alpha_{k}+\alpha_{k-1}\right) \phi_{k}-\alpha_{k-1} \phi_{k-1}=0 \quad \forall k \in \mathbb{Z} .
$$

We denote these solutions $\tilde{\rho}\left(k, k^{*}\right)$ and $\tilde{\sigma}\left(k, k^{*}\right)$, and as fundamental solutions they satisfy

$$
\tilde{\rho}\left(k^{*}, k^{*}\right)=1, \quad \tilde{\rho}\left(k^{*}+1, k^{*}\right)=0, \quad \tilde{\sigma}\left(k^{*}, k^{*}\right)=0, \quad \tilde{\sigma}\left(k^{*}+1, k^{*}\right)=1 .
$$

A general method for finding $\tilde{\rho}\left(k, k^{*}\right)$ and $\tilde{\sigma}\left(k, k^{*}\right)$ is presented in Lemma 3.3 of section 3.3, and we use those results to state the solutions for the single defect case. Define $\tau=\alpha_{0} / \alpha$ and $\nu=\left(1+\alpha+\alpha_{0}\right) / \alpha$. For $k^{*}=0$, when the front is at the defect, the fundamental solutions are

$$
\begin{aligned}
& \tilde{\rho}(k, 0)= \begin{cases}\tau \rho(k), & k>0, \\
\nu \rho(k+1)-\rho(k+2), & k<0,\end{cases} \\
& \tilde{\sigma}(k, 0)= \begin{cases}\nu \sigma(k-1)-\sigma(k-2), & k>0, \\
\tau \sigma(k), & k<0,\end{cases}
\end{aligned}
$$

where $\rho(k)$ and $\sigma(k)$ are defined in (3.12). When the interface lies to the left or right of the defect

$$
\tilde{\rho}\left(k, k^{*}\right)= \begin{cases}\rho(k-1) \theta\left(1, k^{*}\right)+\sigma(k-1) \theta\left(2, k^{*}\right), & k>0, k^{*}<0, \\ \rho\left(k-k^{*}\right), & k \leqslant 0, k^{*}<0, \quad \text { or } \quad k>0, k^{*}>0, \\ \rho(k+1) \theta\left(-1, k^{*}\right)+\sigma(k+1) \theta\left(0, k^{*}\right), & k \leqslant 0, k^{*}>0,\end{cases}
$$

and

$$
\tilde{\sigma}\left(k, k^{*}\right)= \begin{cases}\rho(k-1) \zeta\left(1, k^{*}\right)+\sigma(k-1) \zeta\left(2, k^{*}\right), & k>0, k^{*}<0, \\ \sigma\left(k-k^{*}\right), & k \leqslant 0, k^{*}<0, \quad \text { or } \quad k>0, k^{*}>0, \\ \rho(k+1) \zeta\left(-1, k^{*}\right)+\sigma(k+1) \zeta\left(0, k^{*}\right), & k \leqslant 0, k^{*}>0,\end{cases}
$$

where

$$
\begin{aligned}
\theta\left(1, k^{*}\right) & =\frac{1}{\tau}\left(\nu \rho\left(-k^{*}\right)-\rho\left(-1-k^{*}\right)\right), \\
\theta\left(2, k^{*}\right) & =\frac{1}{\tau}\left(\left(\nu^{2}-\tau^{2}\right) \rho\left(-k^{*}\right)-\nu \rho\left(-1-k^{*}\right)\right), \\
\zeta\left(1, k^{*}\right) & =\frac{1}{\tau}\left(\nu \sigma\left(-k^{*}\right)-\sigma\left(-1-k^{*}\right)\right), \\
\zeta\left(2, k^{*}\right) & =\frac{1}{\tau}\left(\left(\nu^{2}-\tau^{2}\right) \sigma\left(-k^{*}\right)-\nu \sigma\left(-1-k^{*}\right)\right), \\
\theta\left(0, k^{*}\right) & =\frac{1}{\tau}\left(\nu \rho\left(1-k^{*}\right)-\rho\left(2-k^{*}\right)\right), \\
\theta\left(-1, k^{*}\right) & =\frac{1}{\tau}\left(\left(\nu^{2}-\tau^{2}\right) \rho\left(1-k^{*}\right)-\nu \rho\left(2-k^{*}\right)\right),
\end{aligned}
$$

Copyright (c) by SIAM. Unauthorized reproduction of this article is prohibited. 


$$
\begin{aligned}
\zeta\left(0, k^{*}\right) & =\frac{1}{\tau}\left(\nu \sigma\left(1-k^{*}\right)-\sigma\left(2-k^{*}\right)\right), \\
\zeta\left(-1, k^{*}\right) & =\frac{1}{\tau}\left(\left(\nu^{2}-\tau^{2}\right) \sigma\left(1-k^{*}\right)-\nu \sigma\left(2-k^{*}\right)\right) .
\end{aligned}
$$

Given the fundamental solutions, it is straightforward to show that the general solution of (3.1) is

$$
\phi_{k}=\phi_{k^{*}} \tilde{\rho}\left(k, k^{*}\right)+\phi_{k^{*}+1} \tilde{\sigma}\left(k, k^{*}\right)+ \begin{cases}-\sum_{j=k^{*}+1}^{k} \frac{1}{\alpha_{j}} \tilde{\sigma}(k, j), & k>k^{*}, \\ 0, & k=k^{*}, \\ \frac{h_{k^{*}}}{\alpha_{k^{*}}} \tilde{\sigma}\left(k, k^{*}\right), & k<k^{*},\end{cases}
$$

for all $k^{*} \in \mathbb{Z}$. To find an explicit form for (3.28), we must solve for the quantities $\phi_{k^{*}}$ and $\phi_{k^{*}+1}$. We begin with the special case $k^{*}=0$, and consider $k^{*} \neq 0$ in section 3.2 .2 .

3.2.1. Fronts at the defect. Consider $\phi_{k}$ given by (3.28) in the case $k^{*}=0$. Following the approach of section 3.1, we find expressions for $\phi_{0}$ and $\phi_{1}$ which yield solutions that satisfy the boundary conditions (3.2). Using the fundamental solutions $(3.25)$ for $k<0,(3.28)$ reduces to

$$
\begin{aligned}
\phi_{k}= & \frac{\lambda^{-k}}{\lambda-\lambda^{-1}}\left(\phi_{0}\left(\nu-\lambda^{-1}\right)-\tau\left(\phi_{1}+\frac{h_{0}}{\alpha_{0}}\right)\right) \\
& +\frac{\lambda^{k}}{\lambda-\lambda^{-1}}\left(\phi_{0}(\lambda-\nu)+\tau\left(\phi_{1}+\frac{h_{0}}{\alpha_{0}}\right)\right),
\end{aligned}
$$

where $\lambda, \alpha$, and $\mu$ are related by (3.8) and (3.11). Hence, to satisfy the boundary conditions (3.2) as $k \rightarrow-\infty$, we require

$$
\phi_{0}\left(\nu-\lambda^{-1}\right)-\tau\left(\phi_{1}+\frac{h_{0}}{\alpha_{0}}\right)=0 \quad \Longleftrightarrow \quad \phi_{0}(\lambda+\tau-1)-\tau \phi_{1}=\frac{h_{0}}{\alpha},
$$

where we have used the equality $\nu=\frac{1}{\alpha}\left(1+\alpha+\alpha_{0}\right)=2 \mu-1+\frac{\alpha_{0}}{\alpha}=\lambda+\lambda^{-1}+\tau-1$. Likewise, for $k>0$, we use the identity $\nu \sigma(k-1)-\sigma(k-2)=\frac{\lambda^{-k}}{\lambda-\lambda^{-1}}\left(\lambda^{2}-\nu \lambda\right)-$ $\frac{\lambda^{k}}{\lambda-\lambda^{-1}}\left(\lambda^{-2}-\nu \lambda^{-1}\right)$ to write the solution (3.28) in the form

$$
\begin{aligned}
\phi_{k}= & \frac{\lambda^{-k}}{\lambda-\lambda^{-1}}\left(\phi_{1}\left(\lambda^{2}-\nu \lambda\right)+\tau \phi_{0} \lambda+\sum_{j=1}^{k} \frac{\lambda^{j}}{\alpha}\right) \\
& \quad-\frac{\lambda^{k}}{\lambda-\lambda^{-1}}\left(\phi_{1}\left(\lambda^{-2}-\nu \lambda^{-1}\right)+\tau \phi_{0} \lambda^{-1}+\sum_{j=1}^{k} \frac{\lambda^{-j}}{\alpha}\right),
\end{aligned}
$$

and to satisfy (3.2) as $k \rightarrow+\infty$ we require

$$
\phi_{1}\left(\nu-\lambda^{-1}\right)-\tau \phi_{0}-\frac{\lambda}{\alpha} \sum_{j=1}^{\infty} \lambda^{-j}=0 \quad \Longleftrightarrow \quad \phi_{1}(\lambda+\tau-1)-\tau \phi_{0}=\lambda-1
$$

where we have used the identity (3.17). Then solving (3.30), (3.31) for $\phi_{0}$ and $\phi_{1}$ gives

$$
\phi_{0}=v_{1}+h_{0}\left(1-\lambda^{-1}\right) w_{1} \quad \text { and } \quad \phi_{1}=w_{1}+h_{0}\left(1-\lambda^{-1}\right) v_{1}
$$

Copyright (c) by SIAM. Unauthorized reproduction of this article is prohibited. 
where we have defined

$$
v_{1}=\frac{\tau}{\lambda+2 \tau-1} \quad \text { and } \quad w_{1}=\frac{(\lambda+\tau-1)}{\lambda+2 \tau-1},
$$

which satisfy the symmetry property $v_{1}+w_{1}=1$.

Substituting the expressions (3.32) into (3.28) yields the solution

$$
\phi_{k}= \begin{cases}1+\left(\phi_{1}-1\right) \lambda^{1-k}, & k>0, \\ \phi_{0} \lambda^{k}, & k \leqslant 0,\end{cases}
$$

to (3.1) with $k^{*}=0$ and $\phi_{0}$ and $\phi_{1}$ defined by (3.32). Notice that (3.34) has the same form as (3.21), with the difference in the solutions arising only from the different definitions of $\phi_{0}$ and $\phi_{1}$ in (3.32) and (3.19). If $0<\xi^{*}<1$, then $h_{0}=0,(3.32)$ simplifies, and the solution (3.34) becomes

$$
\phi_{k}= \begin{cases}1-v_{1} \lambda^{1-k}, & k>0 \\ v_{1} \lambda^{k}, & k \leqslant 0\end{cases}
$$

One of our primary concerns is understanding what causes a traveling front to become a stationary front. In this respect, the solution (3.35) is of substantial importance, which will be made clear as we more closely consider the parameter values that yield stationary fronts. Hence, we turn our attention to the interval of propagation failure.

If $0<\xi^{*}<1$, then $\phi_{0}<a<\phi_{1}$, and since $h_{0}=0$ in this case, we have $a \in\left(v_{1}, w_{1}\right)$ for $v_{1}$ and $w_{1}$ given in (3.33). So, the interval of propagation failure is simply $\left(v_{1}, w_{1}\right)$ for $0<\xi^{*}<1$. On the other hand, if $\xi^{*}=0$, then $h_{0}=[0,1]$; hence $a=\phi_{0} \in v_{1}+h_{0}\left(1-\lambda^{-1}\right) w_{1}$ or, equivalently, $a \in\left[v_{1}, 1-w_{1} \lambda^{-1}\right]$, and the interval of propagation failure is $\left[v_{1}, 1-w_{1} \lambda^{-1}\right]$ for $\xi^{*}=0$. As one should expect, the interval of propagation failure changes as $\xi^{*}$ changes. By varying $\xi^{*}$, we may derive the interval of propagation failure for fronts that are either to the left or to the right of the defect.

3.2.2. Fronts to the left or right of the defect. Using the general solution (3.28) along with the appropriate fundamental solutions (3.26)-(3.27), we can find expressions for $\phi_{k^{*}}$ and $\phi_{k^{*}+1}$ for any $k^{*}$. This in turn allows us to determine which values of $a$ yield stationary fronts. Rather than following a step-by-step derivation of these results for this special case, we use the general formulae of Theorem 3.6 in section 3.3.2. The proof of that theorem shows how these results are derived.

Suppose $k^{*}<0$. Assuming $\xi^{*}=k^{*}$ implies $a \in \phi_{k^{*}}$ for $h_{k^{*}}=[0,1]$. Thus, the interval of propagation failure is

$$
\left[\frac{1}{\lambda+1}\left(1-\lambda^{2 k^{*}}\left(w_{1}-\lambda v_{1}\right)\right), \frac{\lambda}{\lambda+1}\left(1-\lambda^{2\left(k^{*}-1\right)}\left(w_{1}-\lambda v_{1}\right)\right)\right] \quad \text { for } \xi^{*}=k^{*} .
$$

If instead $k^{*}<\xi^{*}<k^{*}+1$, then $h_{k^{*}}=0$, and the interval of propagation failure is

$$
\left(\frac{1}{\lambda+1}\left[1-\lambda^{2 k^{*}}\left(w_{1}-\lambda v_{1}\right)\right], \frac{\lambda}{\lambda+1}\left[1-\lambda^{2 k^{*}}\left(w_{1}-\lambda v_{1}\right)\right]\right) \quad \text { for } k^{*}<\xi^{*}<k^{*}+1 \text {. }
$$

Now suppose $k^{*}>0$. If $\xi^{*}=k^{*}$, then $a \in \phi_{k^{*}}$ for $h_{k^{*}}=[0,1]$, and the interval of propagation failure is

$$
\left[\frac{1}{\lambda+1}\left(1+\lambda^{1-2 k^{*}}\left(w_{1}-\lambda v_{1}\right)\right), \frac{\lambda}{\lambda+1}\left(1+\lambda^{1-2 k^{*}}\left(w_{1}-\lambda v_{1}\right)\right)\right] \quad \text { for } \xi^{*}=k^{*}
$$



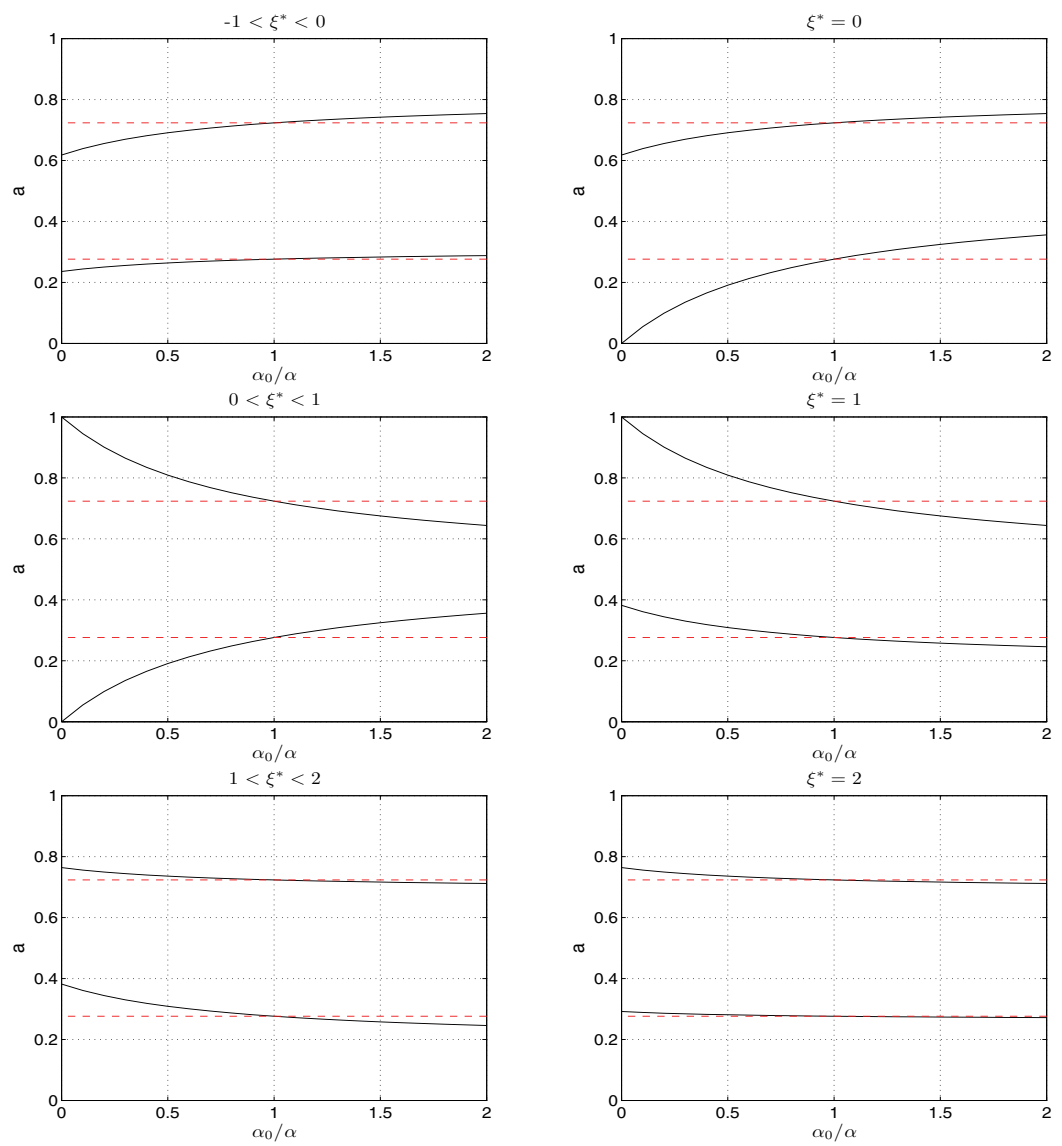

FIG. 1. Solid lines represent the interval of propagation failure against $\tau=\alpha_{0} / \alpha$ with $\alpha=1$ for the case of a single defect. The dashed lines show the interval of propagation failure for $\alpha_{0}=\alpha=1$. Each plot represents a different value of $\xi^{*}$. If a falls between the solid lines in any graph, there exists a stationary front; otherwise we have a traveling front.

But, if $k^{*}<\xi^{*}<k^{*}+1$, then $h_{k^{*}}=0$, and the interval of propagation failure is

$$
\begin{array}{r}
\left(\frac{1}{\lambda+1}\left[1+\lambda^{1-2 k^{*}}\left(w_{1}-\lambda v_{1}\right)\right], \frac{\lambda}{\lambda+1}\left[1+\lambda^{-\left(1+2 k^{*}\right)}\left(w_{1}-\lambda v_{1}\right)\right]\right) \\
\text { for } k^{*}<\xi^{*}<k^{*}+1 .
\end{array}
$$

Thus, we have simultaneously derived explicit formulae for the stationary front forms and the interval of propagation failure in the case of a single defect. Note that the lower boundaries of the intervals of propagation failure when $\xi^{*}=k^{*}$ and $k^{*}<\xi^{*}<k^{*}+1$ agree, while the upper boundary of the interval of propagation failure when $\xi^{*}=k^{*}$ is equal to that of $k^{*}-1<\xi^{*}<k^{*}$. This is clearly seen in Figure 1. In the next section we study how the interval of propagation failure changes with respect to $\alpha_{0}$ and $\xi^{*}$.

3.2.3. Interval of propagation failure. Using the explicit formulae for the interval of propagation failure derived in sections 3.2.1 and 3.2.2, we determine precisely when and where traveling fronts become stationary fronts.

Copyright $@$ by SIAM. Unauthorized reproduction of this article is prohibited. 
THEOREM 3.2. Suppose that $\phi_{k}$ satisfies (3.1) and $\alpha_{k}$ is given by (2.6) with $\mathrm{m}=\mathrm{n}=0$. If $a \in(0,1)$ yields a traveling front when $\alpha_{0}=\alpha$, then there are no corresponding stationary fronts for $\alpha_{0}<\alpha$ and $\xi^{*} \notin(0,1)$, nor for $\alpha_{0}>\alpha$ and $\xi^{*} \in(0,1)$. In addition, there exist $a \in(0,1)$, which yield traveling fronts for $\alpha_{0}=\alpha$ but yield stationary fronts for $\alpha_{0}<\alpha$ and $\xi^{*} \in(0,1)$ or for $\alpha_{0}>\alpha$ and $\xi^{*} \notin(0,1)$.

Proof. First, note that since $\lambda>1$, (3.33) implies that

$$
\frac{d v_{1}}{d \tau}=\frac{\lambda-1}{(\lambda+2 \tau-1)^{2}}>0 \quad \text { and } \quad \frac{d w_{1}}{d \tau}=-\frac{d v_{1}}{d \tau}<0
$$

Hence, $v_{1}$ and $w_{1}$ are respectively monotone increasing and monotone decreasing functions of $\tau=\alpha_{0} / \alpha$.

From section 3.2.2, for all $\xi^{*} \leqslant 0$ and $k^{*}<\xi^{*} \leqslant k^{*}+1$, the upper envelope of the interval of propagation failure is given by

$$
\phi_{k^{*}+1}=\frac{\lambda}{\lambda+1}\left[1-\lambda^{2 k^{*}}\left(w_{1}-\lambda v_{1}\right)\right]=\frac{\lambda}{\lambda+1}\left[1-\lambda^{2 k^{*}} \frac{(1-\tau)(\lambda-1)}{(\lambda+2 \tau-1)}\right] .
$$

Now, using $\frac{d w_{1}}{d \tau}-\lambda \frac{d v_{1}}{d \tau}=\frac{1-\lambda^{2}}{(\lambda+2 \tau-1)^{2}}<0$, we get $\frac{d \phi_{k^{*}+1}}{d \tau}=\frac{\lambda^{1+2 k^{*}}(\lambda-1)}{(\lambda+2 \tau-1)^{2}}>0$, which shows that $\phi_{k^{*}+1}$ is a monotone increasing function of $\tau$. Since

$$
\lim _{k^{*} \rightarrow-\infty} \phi_{k^{*}+1}=w_{0}, \quad \lim _{\tau \rightarrow 1} \phi_{k^{*}+1}=w_{0}, \quad \lim _{\tau \rightarrow+\infty} \phi_{k^{*}+1}=w_{0}\left[1+\frac{1}{2} \lambda^{2 k^{*}}(\lambda-1)\right],
$$

with $w_{0}$ defined by (3.20), we have $\phi_{k^{*}+1}<w_{0}$ when $\alpha_{0}<\alpha$, and $\phi_{k^{*}+1}>w_{0}$ when $\alpha_{0}>\alpha$. If $a \in(0,1)$ yields a traveling front for $\alpha_{0}=\alpha$, meaning $a>w_{0}$, then it is impossible to have $a<\phi_{k^{*}+1}$ when $\tau=\alpha_{0} / \alpha<1$, but if $a \in\left(w_{0}, w_{0}\left[1+\lambda^{2 k^{*}}(\lambda-1) / 2\right]\right)$, then $a<\phi_{k^{*}+1}$ for all $\tau=\alpha_{0} / \alpha$ sufficiently large.

On the other hand, for all $\xi^{*} \geqslant 1$ and $k^{*} \leqslant \xi^{*}<k^{*}+1$ the lower envelope of the interval of propagation failure is given by $\phi_{k^{*}}=\frac{1}{\lambda+1}\left[1+\lambda^{1-2 k^{*}}\left(w_{1}-\lambda v_{1}\right)\right]$ and $\frac{d \phi_{k^{*}}}{d \tau}=\frac{\lambda^{1-2 k^{*}}(1-\lambda)}{(\lambda+2 \tau-1)^{2}}<0$; hence $\phi_{k^{*}}$ is a monotone decreasing function of $\tau$. In this case,

$$
\lim _{k^{*} \rightarrow \infty} \phi_{k^{*}}=v_{0}, \quad \lim _{\tau \rightarrow 1} \phi_{k^{*}}=v_{0}, \quad \lim _{\tau \rightarrow+\infty} \phi_{k^{*}}=v_{0}\left[1-\frac{1}{2} \lambda^{1-2 k^{*}}(\lambda-1)\right],
$$

with $v_{0}$ defined by (3.20), and we find that $\phi_{k^{*}}>v_{0}$ when $\alpha_{0}<\alpha$, and $\phi_{k^{*}}<v_{0}$ when $\alpha_{0}>\alpha$. Hence, if $a$ yields a traveling front for $\alpha_{0}=\alpha$, meaning $a<v_{0}$, then it is impossible to have $a>\phi_{k^{*}}$ when $\alpha_{0}<\alpha$, but if $a \in\left(v_{0}\left[1-\lambda^{1-2 k^{*}}(\lambda-1) / 2\right], v_{0}\right)$, then $a>\phi_{k^{*}}$ for all $\tau=\alpha_{0} / \alpha$ sufficiently large.

Finally, for $0<\xi^{*}<1$ we have $\phi_{k^{*}}=v_{1}$ and $\phi_{k^{*}+1}=w_{1}$. Since $v_{0}=v_{1}$ and $w_{0}=w_{1}$ when $\alpha=\alpha_{0}$, by (3.36), for $\tau=\alpha_{0} / \alpha>1$ we have $v_{1}>v_{0}$ and $w_{1}<w_{0}$, and so it is not possible to have $a \in\left(v_{1}, w_{1}\right)$ whenever $a \notin\left(v_{0}, w_{0}\right)$. However, if $\tau=\alpha_{0} / \alpha<1$, then $v_{1}=\frac{2 \alpha_{0}}{1+4 \alpha_{0}+\sqrt{1+4 \alpha}}<\frac{2 \alpha}{1+4 \alpha+\sqrt{1+4 \alpha}}=v_{0}<w_{0}<w_{1}$, where $v_{0}+w_{0}=1$ and $v_{1}+w_{1}=1$. Thus $a \in\left(v_{1}, v_{0}\right)$ or $a \in\left(w_{0}, w_{1}\right)$ yields traveling fronts when $\alpha=\alpha_{0}$ but stationary fronts for $\alpha_{0}<\alpha$. This completes the proof.

Since, according to $a \in\left[v_{0}, w_{0}\right]$, the interval of propagation failure is $[1 /(\lambda+1)$, $\lambda /(\lambda+1)]$ when $\alpha_{0}=\alpha$, the theorem leads naturally to the following conclusion. Traveling fronts which become pinned, due to a low wave speed relative to the size of $\alpha_{0}<\alpha$, are always pinned in the defect region $0<\xi^{*}<1$ or, equivalently, when $a \in\left(v_{1}, w_{1}\right)$. This is demonstrated in Figure 1 , where the interval of propagation 
failure is plotted as a function of $\tau$ with $\alpha=1$. Values of $a$ that fall between the curves in any given plot yield a stationary front. Note that intervals of propagation failure are closed in plots with $\xi^{*} \in \mathbb{Z}$, while the intervals are open when $\xi^{*} \notin \mathbb{Z}$. Hence, if $a$ falls on a curve, then the result is a stationary front, provided that $a$ also coincides with a lattice point, but if $a$ lies outside the curves, we expect traveling fronts.

Figure 1 shows plots for several values of $k^{*}$, giving a clear picture of how the interval changes as the position of the front relative to the defect changes. The plot for $0<\xi^{*}<1$ merits particular attention, because it corresponds to the wave forms at the defect. This is the only one of the six plots shown which is symmetric about $a=1 / 2$, because fronts are pinned in this interval regardless of the direction from which they approach the defect. We also notice that the length of the interval of propagation failure increases as $\alpha_{0}$ decreases, and $\alpha_{0} \rightarrow 0$ implies that all values of $a$ yield a stationary front.
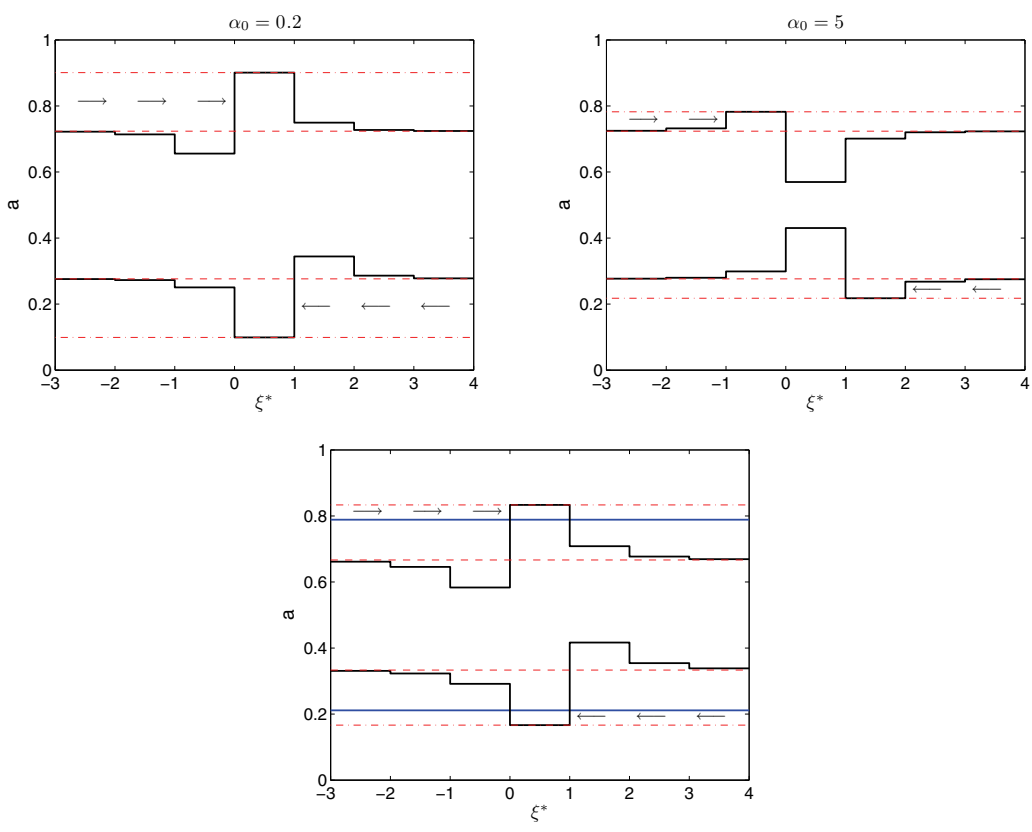

FIG. 2. The interval of propagation failure as a function of $\xi^{*}$. The dashed lines represent the interval of propagation failure when $\alpha_{k}=\alpha$ for all $k$, and the chain lines show the bounds on the interval of propagation failure when defects are present. Arrows show the directions fronts travel for particular values of a. Top left: $\alpha_{0}=0.2, \alpha=1$. Top right: $\alpha_{0}=5, \alpha=1$. Bottom: The solid lines give the interval of propagation failure in two cases, (1) $\alpha_{0}=\alpha=1 / 2$ and (2) $\alpha_{0}=1 / 2$, $\alpha=2$.

Figure 2 demonstrates the results of Theorem 3.2. Choosing $k^{*}<0$ yields a stationary front or a traveling front, depending on the value of $a$, to the left of the defect. Due to (2.9)-(2.10) along with (3.14), increasing this value of $k^{*}$ places the wave form farther to the right with each increment. We also know that fronts traveling with a positive wave speed, corresponding to $a>w_{0}$, travel to the right. Figure 2 shows that a front traveling to the right will not become a stationary front until it reaches the interval $0<\xi^{*}<1$ if $\alpha_{0}<\alpha$. The same is true of fronts traveling to the left, in which case $a<v_{0}$. On the other hand, if $\alpha_{0}>\alpha$, then fronts traveling 
in either direction may get pinned prior to reaching the interval $0<\xi^{*}<1$. In this case, the closer $a$ is to the boundary of the interval of propagation failure defined by the homogeneous diffusion case, the sooner it will get pinned.

It is completely expected that holding $\alpha$ fixed and reducing $\alpha_{0}$ towards zero results in traveling fronts that become pinned. The surprising part of Theorem 3.2 is that $\tau=\alpha_{0} / \alpha$ must decrease in order to pin a front in the interval $[0,1]$, and this can be achieved equally well by holding $\alpha_{0}$ constant and increasing $\alpha$. This is illustrated in the bottom panel of Figure 2, showing that there are values of $a$ for which there are traveling fronts when $\alpha_{0}=\alpha=1 / 2$, but those same values of $a$ yield a stationary front for $\xi^{*} \in(0,1)$ when $\alpha_{0}=1 / 2$ and $\alpha=2$, and the two cases $\alpha_{0}=\alpha=1 / 2$ and $\alpha_{0}=\alpha=2$ both have smaller intervals of propagation failure than $\alpha_{0}=1 / 2, \alpha=2$ for $\xi^{*} \in(0,1)$.

3.3. Inhomogeneous diffusion: Multiple defects. Consider the difference equation (3.1) and allow the diffusion coefficients to vary on an interval of arbitrary length centered about $k=0$ such that $\alpha_{k}$ is given by (2.6). To solve (3.1), we begin by finding the fundamental solutions, denoted $\tilde{\rho}\left(k, k^{*}\right)$ and $\tilde{\sigma}\left(k, k^{*}\right)$, which form the general solution of (3.24). Defining

$$
p_{k}:=1+\alpha_{k}+\alpha_{k-1} \quad \text { and } \quad q_{k}:=\frac{1}{\alpha_{k}}
$$

allows (3.24) to be written as

$$
\phi_{k+1}=q_{k}\left(p_{k} \phi_{k}-\alpha_{k-1} \phi_{k-1}\right) \quad \text { or } \quad \phi_{k-1}=q_{k-1}\left(p_{k} \phi_{k}-\alpha_{k} \phi_{k+1}\right),
$$

which are used to find $\tilde{\rho}\left(k, k^{*}\right)$ and $\tilde{\sigma}\left(k, k^{*}\right)$.

Lemma 3.3. The fundamental solutions, denoted $\tilde{\rho}\left(k, k^{*}\right)$ and $\tilde{\sigma}\left(k, k^{*}\right)$, of (3.24) with (2.6) are given by $\tilde{\rho}\left(k, k^{*}\right)=\rho\left(k-k^{*}\right)$ and $\tilde{\sigma}\left(k, k^{*}\right)=\sigma\left(k-k^{*}\right)$ if $k^{*}<-\mathrm{m}$ and $k \leqslant-\mathrm{m}$ or if $k^{*}>\mathrm{n}$ and $k \geqslant \mathrm{n}$. Otherwise, the fundamental solutions are given by

$$
\tilde{\rho}\left(k, k^{*}\right)= \begin{cases}\rho(k-\mathrm{n}-1) \theta\left(\mathrm{n}+1, k^{*}\right)+\sigma(k-\mathrm{n}-1) \theta\left(\mathrm{n}+2, k^{*}\right), & k>\mathrm{n}, \\ \theta\left(k, k^{*}\right), & -\mathrm{m} \leqslant k \leqslant \mathrm{n}, \\ \rho(k+\mathrm{m}+1) \theta\left(-\mathrm{m}-1, k^{*}\right)+\sigma(k+\mathrm{m}+1) \theta\left(-\mathrm{m}, k^{*}\right), & k<-\mathrm{m},\end{cases}
$$

and

$$
\tilde{\sigma}\left(k, k^{*}\right)= \begin{cases}\rho(k-\mathrm{n}-1) \zeta\left(\mathrm{n}+1, k^{*}\right)+\sigma(k-\mathrm{n}-1) \zeta\left(\mathrm{n}+2, k^{*}\right), & k>\mathrm{n}, \\ \zeta\left(k, k^{*}\right), & -\mathrm{m} \leqslant k \leqslant \mathrm{n}, \\ \rho(k+\mathrm{m}+1) \zeta\left(-\mathrm{m}-1, k^{*}\right)+\sigma(k+\mathrm{m}+1) \zeta\left(-\mathrm{m}, k^{*}\right), & k<-\mathrm{m},\end{cases}
$$

such that $\rho(k)$ and $\sigma(k)$ are given in $(3.12)$ and $\theta\left(k, k^{*}\right)$ and $\zeta\left(k, k^{*}\right)$ are found iteratively by the relations (3.38) with initial conditions

$$
\theta\left(k^{*}, k^{*}\right)=1, \quad \theta\left(k^{*}+1, k^{*}\right)=0, \quad \zeta\left(k^{*}, k^{*}\right)=0, \quad \zeta\left(k^{*}+1, k^{*}\right)=1 .
$$

Proof. Introduce the variable $\psi_{k}=\phi_{k-1}$ and use (3.37) to write (3.38) as

$$
\begin{gathered}
\left(\begin{array}{c}
\psi_{k+1} \\
\phi_{k+1}
\end{array}\right)=\mathbf{M}_{k}\left(\begin{array}{c}
\psi_{k} \\
\phi_{k}
\end{array}\right) \text { and }\left(\begin{array}{c}
\psi_{k-1} \\
\phi_{k-1}
\end{array}\right)=\mathbf{M}_{k}^{-1}\left(\begin{array}{c}
\psi_{k} \\
\phi_{k}
\end{array}\right) \\
\text { with } \mathbf{M}_{k}:=q_{k}\left(\begin{array}{cc}
0 & \alpha_{k} \\
-\alpha_{k-1} & p_{k}
\end{array}\right) .
\end{gathered}
$$

Copyright $\odot$ by SIAM. Unauthorized reproduction of this article is prohibited. 
Using this formulation and results of Teschl [32], the fundamental solutions can be found iteratively by

$$
\tilde{\Phi}\left(k, k^{*}\right):=\left(\begin{array}{cc}
\tilde{\rho}\left(k, k^{*}\right) & \tilde{\sigma}\left(k, k^{*}\right) \\
\tilde{\rho}\left(k+1, k^{*}\right) & \tilde{\sigma}\left(k+1, k^{*}\right)
\end{array}\right)= \begin{cases}\prod_{j=k^{*}+1}^{k} \mathbf{M}_{j}, & k>k^{*}, \\
\mathbf{I}, & k=k^{*}, \\
\prod_{j=k^{*}}^{k+1} \mathbf{M}_{j}^{-1}, & k<k^{*},\end{cases}
$$

such that $\prod_{j=k^{*}+1}^{k} \mathbf{M}_{j}=\mathbf{M}_{k} \cdots \mathbf{M}_{k^{*}+1}$. However, $\mathbf{M}_{k}=\left(\begin{array}{cc}0 & 1 \\ -1 & 2 \mu\end{array}\right):=\mathbf{M}$ for $k<-\mathbf{m}$ or $k>\mathrm{n}$, and

$$
\Phi\left(k-k^{*}\right):=\left(\begin{array}{cc}
\rho\left(k-k^{*}\right) & \sigma\left(k-k^{*}\right) \\
\rho\left(k+1-k^{*}\right) & \sigma\left(k+1-k^{*}\right)
\end{array}\right)= \begin{cases}\mathbf{M}^{k-k^{*}}, & k \neq k^{*}, \\
\mathbf{I}, & k=k^{*},\end{cases}
$$

for $\rho\left(k-k^{*}\right)$ and $\sigma\left(k-k^{*}\right)$ given in (3.12). This naturally implies $\tilde{\rho}\left(k, k^{*}\right)=\rho\left(k-k^{*}\right)$ and $\tilde{\sigma}\left(k, k^{*}\right)=\sigma\left(k-k^{*}\right)$ if $k^{*}<-\mathrm{m}$ and $k \leqslant-\mathrm{m}$ or if $k^{*}>\mathrm{n}$ and $k \geqslant \mathrm{n}$. Otherwise, we can write a general formula for $\tilde{\Phi}\left(k, k^{*}\right)$ in terms of $\rho_{k}$ and $\sigma_{k}$ in the following way:

$$
\tilde{\Phi}\left(k, k^{*}\right)= \begin{cases}\mathbf{M}^{k-\mathrm{n}-1} \prod_{j=k^{*}+1}^{\mathrm{n}+1} \mathbf{M}_{j}, & \mathrm{n}<k \\ \Psi\left(k, k^{*}\right), & -\mathrm{m} \leqslant k \leqslant \mathrm{n}, \\ \mathbf{M}^{k+\mathrm{m}+1} \prod_{j=k^{*}}^{-\mathrm{m}} \mathbf{M}_{j}^{-1}, & k<-\mathrm{m},\end{cases}
$$

where we have defined

$$
\Psi\left(k, k^{*}\right):=\left(\begin{array}{cc}
\theta\left(k, k^{*}\right) & \zeta\left(k, k^{*}\right) \\
\theta\left(k+1, k^{*}\right) & \zeta\left(k+1, k^{*}\right)
\end{array}\right)= \begin{cases}\prod_{j=k^{*}+1}^{k} \mathbf{M}_{j}, & k^{*}<k \leqslant \mathrm{n}, \\
\mathbf{I}, & k=k^{*}, \\
\prod_{j=k^{*}}^{k+1} \mathbf{M}_{j}^{-1}, & -\mathrm{m} \leqslant k<k^{*} .\end{cases}
$$

Thus, for $-\mathrm{m} \leqslant k \leqslant \mathrm{n}$ we have $\tilde{\rho}\left(k, k^{*}\right)=\theta\left(k, k^{*}\right)$ and $\tilde{\sigma}\left(k, k^{*}\right)=\zeta\left(k, k^{*}\right)$. To get $\tilde{\rho}\left(k, k^{*}\right)$ and $\tilde{\sigma}\left(k, k^{*}\right)$ for $k<-\mathrm{m}$ or $k>\mathrm{n}$ we simply use

$$
\tilde{\Phi}\left(k, k^{*}\right)= \begin{cases}\Phi(k-\mathrm{n}-1) \Psi\left(\mathrm{n}+1, k^{*}\right), & k>\mathrm{n}, \\ \Phi(k+\mathrm{m}+1) \Psi\left(-\mathrm{m}-1, k^{*}\right), & k<-\mathrm{m},\end{cases}
$$

and writing these matrices in component form completes the proof.

As in the case of a single defect, the solutions of (3.1) depend on $\left\lfloor\xi^{*}\right\rfloor=k^{*}$, which defines the position of the front relative to the defect. As a result, we derive fundamental solutions that depend on $k^{*}$, and thereby make the statement of the final solution simpler and the interval of propagation failure more accessible. Due to this $k^{*}$-dependence, we must consider two cases: (1) the interface lies in the defect region, (2) the interface lies outside the defect region.

3.3.1. Fronts inside the defect region. Now consider fronts for which $k^{*} \in$ $[-\mathrm{m}, \mathrm{n}]$. We can use the fundamental solutions to find the general solution of (3.1), (2.6), but first we introduce some notation. Using (3.12), the fundamental solutions for $k>\mathrm{n}$ can be written as

$$
\begin{aligned}
& \tilde{\rho}\left(k, k^{*}\right)=\frac{1}{\lambda-\lambda^{-1}}\left[\lambda^{-k} \Gamma^{+}\left(\mathrm{n}, k^{*}\right)-\lambda^{k} \Gamma^{-}\left(\mathrm{n}, k^{*}\right)\right], \\
& \tilde{\sigma}\left(k, k^{*}\right)=\frac{1}{\lambda-\lambda^{-1}}\left[\lambda^{-k} \Lambda^{+}\left(\mathrm{n}, k^{*}\right)-\lambda^{k} \Lambda^{-}\left(\mathrm{n}, k^{*}\right)\right],
\end{aligned}
$$

Copyright $@$ by SIAM. Unauthorized reproduction of this article is prohibited. 
where we have defined

$$
\begin{aligned}
& \Gamma^{ \pm}\left(\mathrm{n}, k^{*}\right):=\theta\left(\mathrm{n}+1, k^{*}\right) \lambda^{ \pm(\mathrm{n}+2)}-\theta\left(\mathrm{n}+2, k^{*}\right) \lambda^{ \pm(\mathrm{n}+1)}, \\
& \Lambda^{ \pm}\left(\mathrm{n}, k^{*}\right):=\zeta\left(\mathrm{n}+1, k^{*}\right) \lambda^{ \pm(\mathrm{n}+2)}-\zeta\left(\mathrm{n}+2, k^{*}\right) \lambda^{ \pm(\mathrm{n}+1)} .
\end{aligned}
$$

For $k<-\mathrm{m}$ we get

$$
\begin{aligned}
& \tilde{\rho}\left(k, k^{*}\right)=\frac{1}{\lambda-\lambda^{-1}}\left[\lambda^{-k} \Gamma^{-}\left(-\mathrm{m}, k^{*}\right)-\lambda^{k} \Gamma^{+}\left(-\mathrm{m}, k^{*}\right)\right], \\
& \tilde{\sigma}\left(k, k^{*}\right)=\frac{1}{\lambda-\lambda^{-1}}\left[\lambda^{-k} \Lambda^{-}\left(-\mathrm{m}, k^{*}\right)-\lambda^{k} \Lambda^{+}\left(-\mathrm{m}, k^{*}\right)\right],
\end{aligned}
$$

with

$$
\begin{aligned}
& \Gamma^{ \pm}\left(-\mathrm{m}, k^{*}\right):=\theta\left(-\mathrm{m}-1, k^{*}\right) \lambda^{ \pm \mathrm{m}}-\theta\left(-\mathrm{m}, k^{*}\right) \lambda^{ \pm(\mathrm{m}+1)}, \\
& \Lambda^{ \pm}\left(-\mathrm{m}, k^{*}\right):=\zeta\left(-\mathrm{m}-1, k^{*}\right) \lambda^{ \pm \mathrm{m}}-\zeta\left(-\mathrm{m}, k^{*}\right) \lambda^{ \pm(\mathrm{m}+1)} .
\end{aligned}
$$

The reader is cautioned here to notice that $\Gamma^{ \pm}\left(-\mathrm{m}, k^{*}\right) \neq \Gamma^{ \pm}\left(\mathrm{n}, k^{*}\right)$ and $\Lambda^{ \pm}\left(-\mathrm{m}, k^{*}\right)$ $\neq \Lambda^{ \pm}\left(\mathrm{n}, k^{*}\right)$ when $\mathrm{m}=\mathrm{n}=0$, but this is the case of a single defect, which is considered in section 3.2. Defining

$$
\Omega_{\mathrm{n}}^{\mathrm{m}}\left(k^{*}\right):=\frac{1}{\Gamma^{-}\left(\mathrm{n}, k^{*}\right) \Lambda^{-}\left(-\mathrm{m}, k^{*}\right)-\Gamma^{-}\left(-\mathrm{m}, k^{*}\right) \Lambda^{-}\left(\mathrm{n}, k^{*}\right)},
$$

we arrive at the main result of this section.

TheOrem 3.4. Suppose that $\alpha_{k}$ is given by (2.6) and that $\left\lfloor\xi^{*}\right\rfloor=k^{*} \in[-\mathrm{m}, \mathrm{n}]$. Then the general solution of (3.1) is given by (3.28), where

$$
\phi_{k^{*}}=\Omega_{\mathrm{n}}^{\mathrm{m}}\left(k^{*}\right) \Lambda^{-}\left(-\mathrm{m}, k^{*}\right)\left[\Lambda^{-}\left(\mathrm{n}, k^{*}\right) \frac{h_{k^{*}}}{\alpha_{k^{*}}}-\lambda^{-\mathrm{n}}\left(1-\lambda^{-1}\right)+\sum_{j=k^{*}+1}^{\mathrm{n}} \frac{1}{\alpha_{j}} \Lambda^{-}(\mathrm{n}, j)\right]
$$

and

$$
\begin{gathered}
\phi_{k^{*}+1}=\Omega_{\mathrm{n}}^{\mathrm{m}}\left(k^{*}\right)\left[\Gamma^{-}\left(-\mathrm{m}, k^{*}\right)\left(\lambda^{-\mathrm{n}}\left(1-\lambda^{-1}\right)-\sum_{j=k^{*}+1}^{\mathrm{n}} \frac{1}{\alpha_{j}} \Lambda^{-}(\mathrm{n}, j)\right)\right. \\
\left.-\Gamma^{-}\left(\mathrm{n}, k^{*}\right) \Lambda^{-}\left(-\mathrm{m}, k^{*}\right) \frac{h_{k^{*}}}{\alpha_{k^{*}}}\right]
\end{gathered}
$$

and the fundamental solutions $\tilde{\rho}\left(k, k^{*}\right)$ and $\tilde{\sigma}\left(k, k^{*}\right)$ are given in Lemma 3.3 .

Proof. Following Teschl [32], one can readily show that

$$
\phi_{k}= \begin{cases}-\sum_{j=k^{*}+1}^{k} \frac{1}{\alpha_{j}} \tilde{\sigma}(k, j), & k>k^{*} \\ 0, & k=k^{*} \\ \frac{h_{k^{*}}}{\alpha_{k^{*}}} \tilde{\sigma}\left(k, k^{*}\right), & k<k^{*}\end{cases}
$$

is a particular solution of (3.1), (2.6), and this in turn shows that (3.28) is the general solution, based on Lemma 3.3. (Note that the sum in (3.42) requires solutions outside the defect region.) To solve for the coefficients $\phi_{k^{*}}$ and $\phi_{k^{*}+1}$ we require

$$
\Gamma^{-}\left(\mathrm{n}, k^{*}\right) \phi_{k^{*}}+\Lambda^{-}\left(\mathrm{n}, k^{*}\right) \phi_{k^{*}+1}=\sum_{j=k^{*}+1}^{\mathrm{n}} \frac{1}{\alpha_{j}} \Lambda^{-}(\mathrm{n}, j)-\lambda^{-\mathrm{n}}\left(1-\lambda^{-1}\right)
$$

Copyright (c) by SIAM. Unauthorized reproduction of this article is prohibited. 
in order to satisfy the boundary conditions as $k \rightarrow \infty$, since $|\lambda|>1$. Similarly, we require

$$
\Gamma^{-}\left(\mathrm{m}, k^{*}\right) \phi_{k^{*}}+\Lambda^{-}\left(\mathrm{m}, k^{*}\right) \phi_{k^{*}+1}=-\Lambda^{-}\left(\mathrm{m}, k^{*}\right) \frac{h_{k^{*}}}{\alpha_{k^{*}}}
$$

to satisfy the boundary conditions as $k \rightarrow-\infty$. Solving the system of equations (3.43)-(3.44) for $\phi_{k^{*}}$ and $\phi_{k^{*}+1}$ gives (3.40) and (3.41), and this completes the proof.

Corollary 3.5. Suppose that $\alpha_{k}$ is given by (2.6) and that $k^{*} \in[-\mathrm{m}, \mathrm{n}]$. Then the existence of solutions for (3.1) is guaranteed by the necessary and sufficient conditions (3.3) or (3.4), where $\phi_{k^{*}}$ and $\phi_{k^{*}+1}$ are defined respectively in (3.40) and (3.41).

This result gives us a completely general way of stating the interval of propagation failure, regardless of the size of the defect region and regardless of the individual values of $\alpha_{k}$ at each point in the defect region. Yet, the result of Corollary 3.5 is somewhat restricted by the terms $\theta\left(\cdot, k^{*}\right)$ and $\zeta\left(\cdot, k^{*}\right)$. In order to analyze the interval of propagation failure in more detail, explicit expressions for these terms are required. These can be obtained by choosing values for $\mathrm{m}$ and $\mathrm{n}$ and then performing the appropriate number of iterations using (3.38).

3.3.2. Fronts outside the defect region. Now the fundamental solutions of Lemma 3.3 are used to find the general solution of (3.1) in the case $k^{*} \notin[-\mathrm{m}, \mathrm{n}]$.

TheOrem 3.6. Suppose that $\alpha_{k}$ is given by (2.6) and that $\left\lfloor\xi^{*}\right\rfloor=k^{*} \notin[-\mathrm{m}, \mathrm{n}]$. Then the general solution of (3.1) is given by (3.28), where the following hold:

- If $k^{*}<-\mathrm{m}$, then

$$
\phi_{k^{*}}=\frac{\frac{h_{k^{*}}}{\alpha} \Lambda^{-}\left(\mathrm{n}, k^{*}\right)-\lambda^{-\mathrm{n}}\left(1-\lambda^{-1}\right)+\sum_{j=k^{*}+1}^{\mathrm{n}} \frac{1}{\alpha_{j}} \Lambda^{-}(\mathrm{n}, j)}{\Gamma^{-}\left(\mathrm{n}, k^{*}\right)+\lambda \Lambda^{-}\left(\mathrm{n}, k^{*}\right)}
$$

and

$$
\phi_{k^{*}+1}=\frac{\frac{-h_{k^{*}}}{\alpha} \Gamma^{-}\left(\mathrm{n}, k^{*}\right)-\lambda^{1-\mathrm{n}}\left(1-\lambda^{-1}\right)+\lambda \sum_{j=k^{*}+1}^{\mathrm{n}} \frac{1}{\alpha_{j}} \Lambda^{-}(\mathrm{n}, j)}{\Gamma^{-}\left(\mathrm{n}, k^{*}\right)+\lambda \Lambda^{-}\left(\mathrm{n}, k^{*}\right)} .
$$

- If $k^{*}>\mathrm{n}$, then

$$
\phi_{k^{*}}=\frac{-\Lambda^{-}\left(-\mathrm{m}, k^{*}\right)\left(\frac{h_{k^{*}}}{\alpha}+1-\lambda^{-1}\right)}{\Gamma^{-}\left(-\mathrm{m}, k^{*}\right)+\lambda^{-1} \Lambda^{-}\left(-\mathrm{m}, k^{*}\right)}
$$

and

$$
\phi_{k^{*}+1}=\frac{-\lambda^{-1} \frac{h_{k^{*}}}{\alpha} \Lambda^{-}\left(-\mathrm{m}, k^{*}\right)+\left(1-\lambda^{-1}\right) \Gamma^{-}\left(-\mathrm{m}, k^{*}\right)}{\Gamma^{-}\left(-\mathrm{m}, k^{*}\right)+\lambda^{-1} \Lambda^{-}\left(-\mathrm{m}, k^{*}\right)} .
$$

Clearly, proof of this result follows in the same manner as the proof of Theorem 3.4 , and results analogous to Corollary 3.5 also hold in this case. To find more precise formulae which define the interval of propagation failure, one must find $\theta\left(\cdot, k^{*}\right)$ and $\zeta\left(\cdot, k^{*}\right)$ for given values of $\mathrm{m}$ and $\mathrm{n}$. However, stationary fronts that result from "slow" traveling fronts which reach the defect region with $\alpha_{k}<\alpha$ are entirely represented by Theorem 3.4. This is demonstrated in the following subsection. 


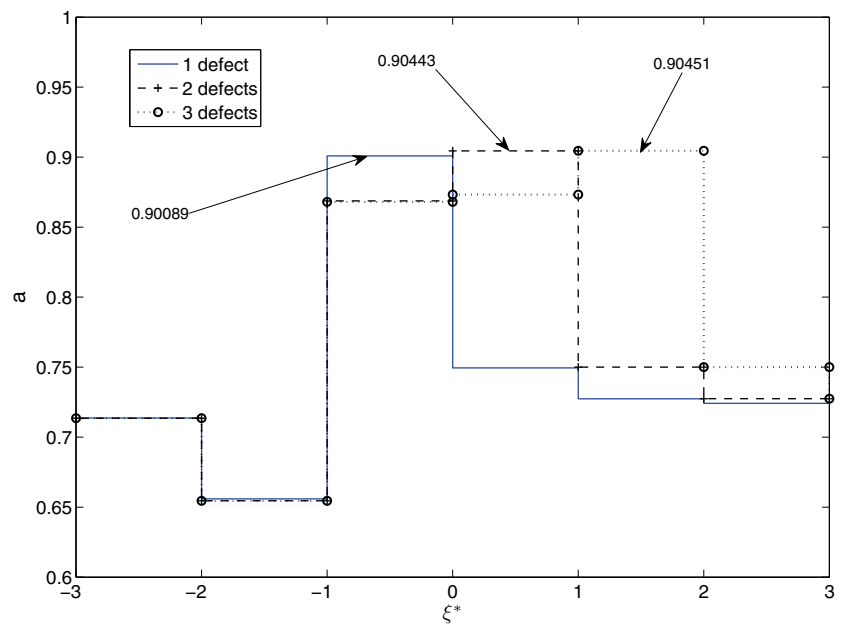

FIG. 3. The upper envelope of the interval of propagation failure as a function of $\xi^{*}$ with $\alpha=1$ and $\alpha_{k}=0.2$ for one, two, and three defects. The arrows indicate the actual values of the upper bound in each of the three cases.

3.3.3. Solutions for multiple defects. An important consideration for cases with multiple defects is the significance of the length of the interval $[-m, n]$. Theorems 3.4 and 3.6 allow us to evaluate the general solution (3.28) to (3.1) when $\alpha_{k}$ is given by (2.6). Figure 3 shows that when $\alpha_{k}$ is held fixed for $k \in[-\mathrm{m}, \mathrm{n}]$ for a defect region of one, two, or three mesh points, the maximum width of the interval of propagation failure is slightly increased with each additional defect and approaches a constant value exponentially fast. (See (3.40) and (3.41).) Hence, increasing the number of defects has little or no effect in determining whether or not a traveling front gets pinned, especially when the number of defects is large. However, increasing the number of defects may have a large effect on where a traveling front is pinned. As the plot shows, for values of $a$ slightly smaller than 0.9 , the traveling front may get pinned in any of the three intervals $(-1,0),(0,1)$, or $(1,2)$, depending on the number of defects.

In Figure 4, the upper envelope of the interval of propagation failure is plotted for three defects as a function of $\xi^{*}$ and $\alpha_{\jmath}$, for $\jmath=-1,0,1$. In each plot, one defect is varied between 0 and 1 , while the others are fixed at $1 / 2$. For particular values of $\alpha_{-1}, \alpha_{0}$, and $\alpha_{1}$ it is possible that a traveling front is pinned in any one of the three intervals, $\xi^{*} \in(-1,0),(0,1),(1,2)$, depending on the wave speed, but the front will not be pinned anywhere outside these three intervals. Also, the interval of propagation failure is always affected by the size of the defect at a later point, and the effects are inversely proportional to the change in that defect. This is noticeable in each plot, where increasing $\alpha_{\jmath}$ implies an increase in the interval of propagation failure for $\xi^{*} \in(\jmath, \jmath+1)$, but a decrease for $\xi^{*} \in(\jmath-1, \jmath)$.

4. Derivation of solutions for nonzero wave speed. We now derive solutions for the case of nonzero wave speed (2.12), by substituting the traveling wave ansatz (2.7) into the evolution equation (2.1), which results in a boundary value problem. Then, taking advantage of the equation's structure, we apply a Fourier transform to derive candidate traveling front solutions. We conclude by checking the consistency 

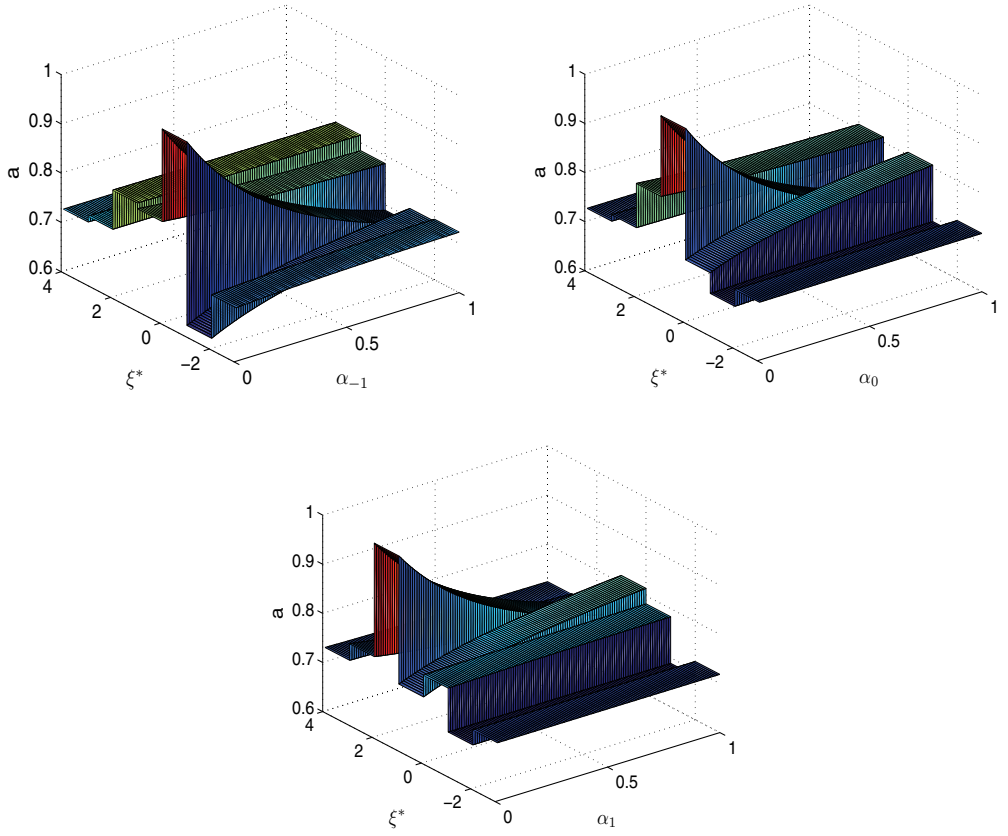

FIG. 4. Upper bound of the interval of propagation failure for three defects and $\alpha=1$. Top left: $\alpha_{0}=\alpha_{1}=0.5$ and $0 \leqslant \alpha_{-1} \leqslant 1$; top right: $\alpha_{-1}=\alpha_{1}=0.5$ and $0 \leqslant \alpha_{0} \leqslant 1$; bottom: $\alpha_{-1}=\alpha_{0}=0.5$ and $0 \leqslant \alpha_{1} \leqslant 1$.

of these solutions with (2.9).

Based on (2.6)-(2.7), we assume that the wave speeds satisfy $c_{j}(t) \rightarrow c t$ as $j \rightarrow$ $\pm \infty$. Thus, we define sets

$$
\begin{gathered}
\mathcal{R}=\left\{j \in \mathbb{Z}: c_{j}(t) \neq c t\right\}, \quad \mathcal{S}=\{j \in \mathbb{Z}: j \in \mathcal{R}, j+1 \in \mathcal{R}, \text { or } j-1 \in \mathcal{R}\} \\
\mathcal{T}=\left\{j \in \mathbb{Z}: \alpha_{j} \neq \alpha\right\}
\end{gathered}
$$

and let $c_{j}(t)=c t$ for $j \notin \mathcal{R}$. Then

$$
\xi_{j}= \begin{cases}j-c_{j}(t), & j \in \mathcal{R}, \\ j-c t, & j \notin \mathcal{R},\end{cases}
$$

and we seek solutions with $\mathcal{R}$ finite.

4.1. Problem set-up. Substituting (2.7) into (2.1) yields

$$
-d_{j} \varphi^{\prime}\left(\xi_{j}\right)=\alpha_{j}\left(\varphi\left(\xi_{j+1}\right)-\varphi\left(\xi_{j}\right)\right)+\alpha_{j-1}\left(\varphi\left(\xi_{j-1}\right)-\varphi\left(\xi_{j}\right)\right)-\varphi\left(\xi_{j}\right)+h\left(\xi_{j}-\xi^{*}\right)
$$

for $j \in \mathbb{Z}$, where we set $d_{j}(t)=\dot{c}_{j}(t)$. Equivalently, we may write (4.3) as

$$
\begin{aligned}
-d_{j} \varphi^{\prime}\left(\xi_{j}\right)= & \alpha\left(\varphi\left(\xi_{j}+1\right)-\varphi\left(\xi_{j}\right)\right)+\alpha\left(\varphi\left(\xi_{j}-1\right)-\varphi\left(\xi_{j}\right)\right)-\varphi\left(\xi_{j}\right)+h\left(\xi_{j}-\xi^{*}\right) \\
& +\left(\alpha_{j}-\alpha\right)\left(\varphi\left(\xi_{j+1}\right)-\varphi\left(\xi_{j}\right)\right)+\left(\alpha_{j-1}-\alpha\right)\left(\varphi\left(\xi_{j-1}\right)-\varphi\left(\xi_{j}\right)\right) \\
& +\alpha\left(\varphi\left(\xi_{j+1}\right)-\varphi\left(\xi_{j}+1\right)\right)+\alpha\left(\varphi\left(\xi_{j-1}\right)-\varphi\left(\xi_{j}-1\right)\right) .
\end{aligned}
$$

Remark. We have omitted the $\xi^{*}$-dependence of $\varphi$ here in order to avoid messy notation, but we must emphasize the importance of $\xi^{*}$ for the following derivation. 
Each $\xi^{*}$ gives a different problem for which the solution is a particular front, which is part of a family of fronts defined by the size of the defect and the wave speed far away from the defect region. With every change of $\xi^{*}$, the wave form, defined by $\varphi\left(\xi ; \xi^{*}\right)$, changes as well as its position in the medium. Hence, variation of the parameter $\xi^{*}$ enables one to study how the family of fronts changes with respect to the medium. This is made evident through the construction (2.8)-(2.10).

The linear system of differential equations (4.4) can be solved exactly, but this requires knowing the values $d_{j}$. In the case of homogeneous diffusion, a wave speed $c$ is chosen, then the solution is obtained, giving the corresponding value of $a$. In this case, we must choose the values $d_{j}$ such that the resulting solution gives the value of $a$ corresponding to $c$ as $j \rightarrow \pm \infty$.

Using (4.1)-(4.2) with $j-c t=\xi \in \mathbb{R}$, we may write (4.4) as

$$
\begin{aligned}
& -c \varphi^{\prime}(\xi)+\sum_{j \in \mathcal{R}} \delta_{j}(\xi)\left(-d_{j}+c\right) \varphi^{\prime}\left(\xi_{j}\right) \\
& =\alpha(\varphi(\xi+1)-2 \varphi(\xi)+\varphi(\xi-1))-\varphi(\xi)+h\left(\xi-\xi^{*}\right) \\
& \quad+\alpha \sum_{j \in \mathcal{S}} \delta_{j}(\xi)\left[\varphi\left(\xi_{j+1}\right)-\varphi\left(\xi_{j}+1\right)+\varphi\left(\xi_{j-1}\right)-\varphi\left(\xi_{j}-1\right)\right] \\
& \quad+\sum_{j \in \mathcal{T}}\left(\alpha_{j}-\alpha\right)\left(\delta_{j}(\xi)-\delta_{j+1}(\xi)\right)\left(\varphi\left(\xi_{j+1}\right)-\varphi\left(\xi_{j}\right)\right),
\end{aligned}
$$

where $\delta_{j}(x)=\delta\left(x-\xi_{j}\right)$ is the Kronecker delta function; then substituting (4.3) into the left-hand side of this expression and defining $\beta_{j}=\left(c / d_{j}\right)-1$ and $\gamma_{j}=\alpha_{j}-\alpha$ gives

$$
\begin{aligned}
& -c \varphi^{\prime}(\xi)=\alpha(\varphi(\xi+1)-2 \varphi(\xi)+\varphi(\xi-1))-\varphi(\xi)+h\left(\xi-\xi^{*}\right) \\
& +\sum_{j \in \mathcal{R}} \beta_{j} \delta_{j}(\xi)\left(\alpha_{j}\left(\varphi\left(\xi_{j+1}\right)-\varphi\left(\xi_{j}\right)\right)+\alpha_{j-1}\left(\varphi\left(\xi_{j-1}\right)-\varphi\left(\xi_{j}\right)\right)-\varphi\left(\xi_{j}\right)+h\left(\xi_{j}-\xi^{*}\right)\right) \\
& +\alpha \sum_{j \in \mathcal{S}} \delta_{j}(\xi)\left[\varphi\left(\xi_{j+1}\right)-\varphi\left(\xi_{j}+1\right)+\varphi\left(\xi_{j-1}\right)-\varphi\left(\xi_{j}-1\right)\right] \\
& +\sum_{j \in \mathcal{T}} \gamma_{j}\left(\delta_{j}(\xi)-\delta_{j+1}(\xi)\right)\left(\varphi\left(\xi_{j+1}\right)-\varphi\left(\xi_{j}\right)\right) .
\end{aligned}
$$

Notice that the first line of this equation is the traveling front problem for the case of homogeneous diffusion [5], and the three lines that follow represent the perturbation, which results from allowing $\mathcal{T} \neq \emptyset$ according to (2.6) and subsequently choosing $\mathcal{R}$ and $\mathcal{S}$ to be nonempty. Hence, we are able to follow the techniques of $[5,12]$ to solve the equation, and before proceeding we need the following preliminary result.

Lemma 4.1. There exists an $\varepsilon>0$ such that $|\varphi(\xi)| \leqslant K e^{\varepsilon \xi}$ for $\xi \leqslant 0$ and some $K>0$.

Proof. For the proof, refer to [5, Lemma 4.1] and [12, Lemma 3.1].

4.2. Candidate solutions via Fourier transform. Since the $\alpha_{j}$ 's are defined only at the lattice points, replace the Kronecker delta $\delta(x)$ with the approximation to the Dirac delta $\delta_{\ell}(x)$, where

$$
\delta_{\ell}(x)= \begin{cases}1 / \ell, & |x| \leqslant \ell / 2 \\ 0, & |x|>\ell / 2 .\end{cases}
$$

Copyright (c) by SIAM. Unauthorized reproduction of this article is prohibited. 
We seek a solution in the sense of distributions, and based upon Lemma 4.1, apply the change of variables $\varphi_{\varepsilon}(\xi)=e^{-\varepsilon \xi} \varphi(\xi)$ for $\varepsilon>0$ sufficiently small. Then $\varphi_{\varepsilon}(\xi)$ satisfies the equation

$$
\begin{gathered}
-c \varphi_{\varepsilon}^{\prime}(\xi)=\alpha\left(e^{\varepsilon} \varphi_{\varepsilon}(\xi+1)-2 \varphi_{\varepsilon}(\xi)+e^{-\varepsilon} \varphi_{\varepsilon}(\xi-1)\right)-(1-c \varepsilon) \varphi_{\varepsilon}(\xi)+e^{-\varepsilon \xi} h\left(\xi-\xi^{*}\right) \\
+\sum_{j \in \mathcal{R}} \beta_{j} \delta_{\ell}\left(\xi-\xi_{j}\right)\left[\alpha_{j}\left(e^{\varepsilon} \varphi_{\varepsilon}\left(\xi_{j+1}\right)-\varphi_{\varepsilon}\left(\xi_{j}\right)\right)+\alpha_{j-1}\left(e^{-\varepsilon} \varphi_{\varepsilon}\left(\xi_{j-1}\right)-\varphi_{\varepsilon}\left(\xi_{j}\right)\right)\right. \\
\left.\quad-\varphi_{\varepsilon}\left(\xi_{j}\right)+e^{-\varepsilon \xi_{j}} h\left(\xi_{j}-\xi^{*}\right)\right] \\
+\sum_{j \in \mathcal{S}} \alpha \delta_{\ell}\left(\xi-\xi_{j}\right)\left[e^{-\varepsilon\left(\xi_{j}-\xi_{j+1}\right)} \varphi_{\varepsilon}\left(\xi_{j+1}\right)-e^{\varepsilon} \varphi_{\varepsilon}\left(\xi_{j}+1\right)+e^{-\varepsilon\left(\xi_{j}-\xi_{j-1}\right)} \varphi_{\varepsilon}\left(\xi_{j-1}\right)\right. \\
\left.-e^{-\varepsilon} \varphi_{\varepsilon}\left(\xi_{j}-1\right)\right] \\
+\sum_{j \in \mathcal{T}} \gamma_{j}\left[\delta_{\ell}\left(\xi-\xi_{j}\right)\left(e^{-\varepsilon\left(\xi_{j}-\xi_{j+1}\right)} \varphi_{\varepsilon}\left(\xi_{j+1}\right)-\varphi_{\varepsilon}\left(\xi_{j}\right)\right)\right. \\
\left.+\delta_{\ell}\left(\xi-\xi_{j+1}\right)\left(e^{-\varepsilon\left(\xi_{j+1}-\xi_{j}\right)} \varphi_{\varepsilon}\left(\xi_{j}\right)-\varphi_{\varepsilon}\left(\xi_{j+1}\right)\right)\right] .
\end{gathered}
$$

Apply a Fourier transform $\widehat{\varphi}_{\varepsilon}(s)=\int_{-\infty}^{\infty} e^{-\mathrm{i} s \xi} \varphi_{\varepsilon}(\xi) d \xi$, and then notice that $\int_{-\infty}^{\infty} e^{-\mathrm{i} s \xi} \varphi_{\varepsilon}^{\prime}(\xi) d \xi=\mathrm{i} s \widehat{\varphi}_{\varepsilon}(s)$, with the use of integration by parts, and $\int_{-\infty}^{\infty} e^{-(\mathrm{i} s+\varepsilon) \xi} h$. $\left(\xi-\xi^{*}\right) d \xi=\int_{\xi^{*}}^{\infty} e^{-(i s+\varepsilon) \xi} d \xi=\frac{e^{-(i s+\varepsilon) \xi^{*}}}{\mathrm{i} s+\varepsilon}$, and $\int_{-\infty}^{\infty} e^{-\mathrm{i} s \xi-\varepsilon} \varphi_{\varepsilon}(\xi-1) d \xi=e^{-(\mathrm{i} s+\varepsilon)} \widehat{\varphi}_{\varepsilon}(s)$. For all terms containing $\delta_{\ell}$, the Fourier transform implies $\int_{-\infty}^{\infty} e^{-i s \xi} \delta_{\ell}\left(\xi-\xi_{k}\right) K d \xi=$ $\frac{K}{\ell} \int_{\xi_{k}-\ell / 2}^{\xi_{k}+\ell / 2} e^{-\mathrm{i} s \xi} d \xi$ for any $K \in \mathbb{R}$ and $k \in \mathbb{Z}$. Then using the mean value theorem for integrals and letting $\ell \rightarrow 0$ gives $\int_{-\infty}^{\infty} e^{-i s \xi} \delta_{\ell}\left(\xi-\xi_{k}\right) K d \xi=K e^{-i s \xi_{k}}$. Thus,

$$
\begin{gathered}
\widehat{\varphi}_{\varepsilon}(s)=\frac{e^{-(\mathrm{i} s+\varepsilon) \xi^{*}}}{(\mathrm{i} s+\varepsilon) R(s-\mathrm{i} \varepsilon)} \\
+\sum_{j \in \mathcal{R}} \frac{\beta_{j} e^{-\mathrm{i} s \xi_{j}}}{R(s-\mathrm{i} \varepsilon)}\left[\alpha_{j}\left(e^{\varepsilon} \varphi_{\varepsilon}\left(\xi_{j+1}\right)-\varphi_{\varepsilon}\left(\xi_{j}\right)\right)+\alpha_{j-1}\left(e^{-\varepsilon} \varphi_{\varepsilon}\left(\xi_{j-1}\right)-\varphi_{\varepsilon}\left(\xi_{j}\right)\right)\right. \\
\left.\quad-\varphi_{\varepsilon}\left(\xi_{j}\right)+e^{-\varepsilon \xi_{j}} h\left(\xi_{j}-\xi^{*}\right)\right] \\
+\sum_{j \in \mathcal{S}} \frac{\alpha e^{-\mathrm{i} s \xi_{j}}}{R(s-\mathrm{i} \varepsilon)}\left[e^{-\varepsilon\left(\xi_{j}-\xi_{j+1}\right)} \varphi_{\varepsilon}\left(\xi_{j+1}\right)-e^{\varepsilon} \varphi_{\varepsilon}\left(\xi_{j}+1\right)+e^{-\varepsilon\left(\xi_{j}-\xi_{j-1}\right)} \varphi_{\varepsilon}\left(\xi_{j-1}\right)\right. \\
+\sum_{j \in \mathcal{T}} \frac{\gamma_{j}}{R(s-\mathrm{i} \varepsilon)}\left[e^{-\mathrm{i} s \xi_{j}}\left(e^{-\varepsilon\left(\xi_{j}-\xi_{j+1}\right)} \varphi_{\varepsilon}\left(\xi_{j+1}\right)-\varphi_{\varepsilon}\left(\xi_{j}\right)\right)\right. \\
\left.+e^{-\mathrm{i} s \xi_{j+1}}\left(e^{-\varepsilon\left(\xi_{j+1}-\xi_{j}\right)} \varphi_{\varepsilon}\left(\xi_{j}\right)-\varphi_{\varepsilon}\left(\xi_{j+1}\right)\right)\right]
\end{gathered}
$$

where we define $R(s):=1-\mathrm{i} c s+2 \alpha(1-\cos (s))$.

Then using the inverse Fourier transform and following [5], we obtain, in the limit as $\varepsilon \rightarrow 0$,

$$
\varphi\left(\xi ; \xi^{*}\right)=\psi\left(\xi ; \xi^{*}\right)+\chi\left(\xi ; \xi^{*}\right)
$$

where

$$
\psi\left(\xi ; \xi^{*}\right)=\frac{1}{2}+\frac{1}{\pi} \int_{0}^{\infty} \frac{A(s) \sin \left(s\left(\xi-\xi^{*}\right)\right)}{s\left(A(s)^{2}+c^{2} s^{2}\right)} d s+\frac{c}{\pi} \int_{0}^{\infty} \frac{\cos \left(s\left(\xi-\xi^{*}\right)\right)}{A(s)^{2}+c^{2} s^{2}} d s
$$

Copyright (c) by SIAM. Unauthorized reproduction of this article is prohibited. 
for $A(s)=1+2 \alpha(1-\cos (s))$ is the solution to the equation with homogeneous diffusion $\alpha_{j}=\alpha$ for all $j \in \mathbb{Z}[5]$. The term $\chi\left(\xi ; \xi^{*}\right)$ represents the perturbation from the homogeneous diffusion problem and takes the form

$$
\chi\left(\xi ; \xi^{*}\right)=\sum_{j \in \mathcal{R}} \beta_{j} F_{j}(\xi) B_{j}\left(\xi^{*}\right)+\alpha \sum_{j \in \mathcal{S}} F_{j}(\xi) C_{j}\left(\xi^{*}\right)+\sum_{j \in \mathcal{T}} \gamma_{j}\left(F_{j}(\xi)-F_{j+1}(\xi)\right) D_{j}\left(\xi^{*}\right),
$$

where $F_{j}(\xi):=\frac{1}{2 \pi} \int_{-\infty}^{\infty} \frac{e^{(i \mathrm{~s}+\varepsilon)\left(\xi-\xi_{j}\right)}}{R(s-\mathrm{i} \varepsilon)} d s$ is the integral that results from the inverse Fourier transform and

$$
\begin{aligned}
& B_{j}\left(\xi^{*}\right)=\alpha_{j}\left(\varphi\left(\xi_{j+1} ; \xi^{*}\right)-\varphi\left(\xi_{j} ; \xi^{*}\right)\right)+\alpha_{j-1}\left(\varphi\left(\xi_{j-1} ; \xi^{*}\right)-\varphi\left(\xi_{j} ; \xi^{*}\right)\right)-\varphi\left(\xi_{j} ; \xi^{*}\right)+h\left(\xi_{j}-\xi^{*}\right) \\
& C_{j}\left(\xi^{*}\right)=\varphi\left(\xi_{j+1} ; \xi^{*}\right)-\varphi\left(\xi_{j}+1 ; \xi^{*}\right)+\varphi\left(\xi_{j-1} ; \xi^{*}\right)-\varphi\left(\xi_{j}-1 ; \xi^{*}\right) \\
& D_{j}\left(\xi^{*}\right)=\varphi\left(\xi_{j+1} ; \xi^{*}\right)-\varphi\left(\xi_{j} ; \xi^{*}\right) .
\end{aligned}
$$

To simplify $F_{j}$, let $\varepsilon \rightarrow 0$ so that

$$
F_{j}(\xi)=\frac{1}{2 \pi} \int_{-\infty}^{\infty} \frac{e^{\mathrm{i} s\left(\xi-\xi_{j}\right)}}{R(s)} d s=\frac{1}{2 \pi} \int_{0}^{\infty} \frac{e^{\mathrm{i} s\left(\xi-\xi_{j}\right)}}{R(s)}+\frac{e^{-\mathrm{i} s\left(\xi-\xi_{j}\right)}}{R(-s)} d s
$$

and the two identities $R(-s) e^{\mathrm{i} s\left(\xi-\xi_{j}\right)}+R(s) e^{-\mathrm{i} s\left(\xi-\xi_{j}\right)}=2 A(s) \cos \left(s\left(\xi-\xi_{j}\right)\right)$ $-2 c s \sin \left(s\left(\xi-\xi_{j}\right)\right)$ and $R(s) R(-s)=A(s)^{2}+c^{2} s^{2}$ imply

$$
F_{j}(\xi)=\frac{1}{\pi} \int_{0}^{\infty} \frac{A(s) \cos \left(s\left(\xi-\xi_{j}\right)\right)-c s \sin \left(s\left(\xi-\xi_{j}\right)\right)}{A(s)^{2}+c^{2} s^{2}} d s .
$$

The terms $\varphi\left(x ; \xi^{*}\right)$, which are contained in $\chi\left(\xi ; \xi^{*}\right)$ according to (4.9), can be found by solving the linear system that results from evaluating (4.6) at $\xi=x$.

In order for (4.6)-(4.10) to represent a solution for all $\xi^{*} \in \mathbb{R}$ the conditions (2.9) must be satisfied with $\varphi\left(\xi^{*}, \xi^{*}\right)=a$. Thus, we must choose $d_{j}$ so that $\chi\left(\xi^{*}, \xi^{*}\right)=0$, and

$$
\psi\left(x ; \xi^{*}\right)+\chi\left(x ; \xi^{*}\right)<a \text { for } x<\xi^{*} \quad \text { and } \quad \psi\left(x ; \xi^{*}\right)+\chi\left(x ; \xi^{*}\right)>a \text { for } x>\xi^{*},
$$

because $\psi\left(\xi^{*}, \xi^{*}\right)=a$. Details on verifying (4.11) and finding $c_{j}$ and $d_{j}$ are discussed in section 4.3. If (4.11) is not satisfied, we may look for waves with multiple crossings of $a$ using the techniques of [13].

Provided that (4.11) is satisfied, we have a family of solutions $\varphi\left(\xi ; \xi^{*}\right)$, which depends on the wave speed far from the defect region, given by $c$, and the size and number of the defects $\alpha_{j}$ relative to $\alpha$. Each value of $\xi^{*} \in \mathbb{R}$ corresponds to a member of this family, and variation of the parameter $\xi^{*}$ changes the distance between the front and the defect region, due to the construction (2.8)-(2.10). In other words, each member of this family of fronts for given $c, \alpha$, and $\alpha_{j}$ has a different position and shape in the media depending on its distance from or place within the defect region. In fact, we are able to change the position of the front in the media by changing $\xi^{*}$, similar to the way $c t$ translates a front defined by $\varphi\left(j-c t ; \xi^{*}\right)$. As a result, each value of $\xi^{*}$ directly corresponds to $c_{j}$, and thereby $d_{j}$, and we estimate $c_{j}\left(\xi^{*}\right)$ and $d_{j}\left(\xi^{*}\right)$ based on the position of the front relative to the defect.

Consider the special case of one defect with $\mathcal{T}=\{0\}$, so that $\alpha_{j}=\alpha$ for all $j \neq 0$, and allow $\alpha_{0} \neq \alpha$. (Cases with $|\mathcal{T}|>1$ may be considered using (4.6)-(4.10) and 
similar arguments.) We begin consideration of this case by allowing the wave speed to vary at only one point (the point of defect) by letting $\mathcal{R}=\{0\}$ and $\mathcal{S}=\{-1,0,1\}$. To state this solution explicitly, one must solve for $B_{0}\left(\xi^{*}\right), C_{j}\left(\xi^{*}\right)$, and $D_{0}\left(\xi^{*}\right)$, given in (4.9), and this is achieved by evaluating

$$
\varphi\left(\xi ; \xi^{*}\right)=\psi\left(\xi ; \xi^{*}\right)+\alpha \sum_{j=-1}^{1} F_{j}(\xi) C_{j}\left(\xi^{*}\right)+\gamma_{0}\left(F_{0}(\xi)-F_{1}(\xi)\right) D_{0}\left(\xi^{*}\right)+\beta_{0} F_{0}(\xi) B_{0}\left(\xi^{*}\right)
$$

at $\xi=x_{j}$, where $x_{j}$ are elements of $\mathbf{x}:=\left[\xi_{0}-1, \xi_{-1}, \xi_{0}, \xi_{1}-1, \xi_{0}+1, \xi_{1}\right]^{T}$, and solving the resulting linear system for $\mathbf{w}:=\left[\varphi\left(\xi_{0}-1\right), \varphi\left(\xi_{-1}\right), \varphi\left(\xi_{0}\right), \varphi\left(\xi_{1}-1\right), \varphi\left(\xi_{0}+1\right)\right.$, $\left.\varphi\left(\xi_{1}\right)\right]^{T}$. Experimentation reveals that these solutions are comparable to numerical solutions of the evolution equation, but we may consider solutions with multiple variable wave speeds. Allowing more wave speeds to depend on the lattice and on time for the case of a single defect, so that $\mathcal{T}=\{0\}$ and $|\mathcal{R}|=r \geqslant 1$, we must solve a linear system in which the dimension of the vector of unknowns is

$$
\operatorname{dim}(\mathbf{w})= \begin{cases}3 r+4, & r \text { even } \\ 3 r+3, & r \text { odd }\end{cases}
$$

Once $c_{j}\left(\xi^{*}\right)$ for $j \in \mathcal{R}$ is determined with sufficient accuracy, we are able to consider the wave forms $\varphi\left(\xi ; \xi^{*}\right)$. At this point it is necessary to check that the conditions (4.11) are satisfied, and this can be done numerically. Since $\varphi\left(\xi ; \xi^{*}\right) \rightarrow \psi\left(\xi ; \xi^{*}\right)$ as $\xi \rightarrow \pm \infty,(4.11)$ is satisfied if the interface is sufficiently far from the defect. Our experimental results verify that the condition is also satisfied near the defect.

4.3. Numerical results. Here, we illustrate the behavior of a front through a single defect. Let $a$ denote the value of the detuning parameter for the traveling front of the problem with homogeneous diffusion $\alpha$ and wave speed $c$. For all experiments we use $\alpha=1$ and $a=0.8116$, which implies $c \approx 1$. The behavior of the solutions (4.6)-(4.10) is demonstrated through numerical integration, using the Filon quadrature method, which is well suited for the highly oscillatory integrals that result from the Fourier transform $[17,18]$. All experiments have been performed using an absolute error tolerance of $10^{-4}$ with a truncated interval of integration $\left[0,10^{3}\right]$. As input we use numerical approximations of the wave speeds $d_{j}$. These are obtained by solving the evolution equation with the Runge-Kutta-Fehlberg method, and we use a minimum error tolerance of $10^{-10}$. To compute the variable wave speed we measure the time needed for points on the interface to travel from one lattice point to the next. For example, if $u_{0}\left(t_{0}\right)=a$ and $u_{1}\left(t_{1}\right)=a$, then the wave speed is estimated to be $d=1 /\left(t_{1}-t_{0}\right)$, and the procedure is repeated at intermediate time steps to increase the accuracy of the estimate.

In Figure 5 we plot wave speed estimates for three values of $\alpha_{0}$. Since $c=1$ in this case, integer values of $t$ directly correspond to lattice points only if $\alpha_{0}=\alpha$. The front with no defects $\left(\alpha_{0}=\alpha\right)$ has $u_{0}=a$ at time $t=13$, but the front with one defect and $\alpha_{0}=0.7$ has $u_{0}=a$ at time $t \approx 14$. This is emphasized in the figure by a large deceleration between times 13 and 14. Though this deceleration is expected, we also notice a significant acceleration that takes place prior to deceleration. This speed-up is less intuitive but is certainly consistent with the results of section 3 , where we saw that the interval of propagation failure always decreased for $\xi^{*}$ in the interval preceding the defect region, but that just inside the defect region the interval of propagation failure showed a dramatic increase. We also point out that the return 


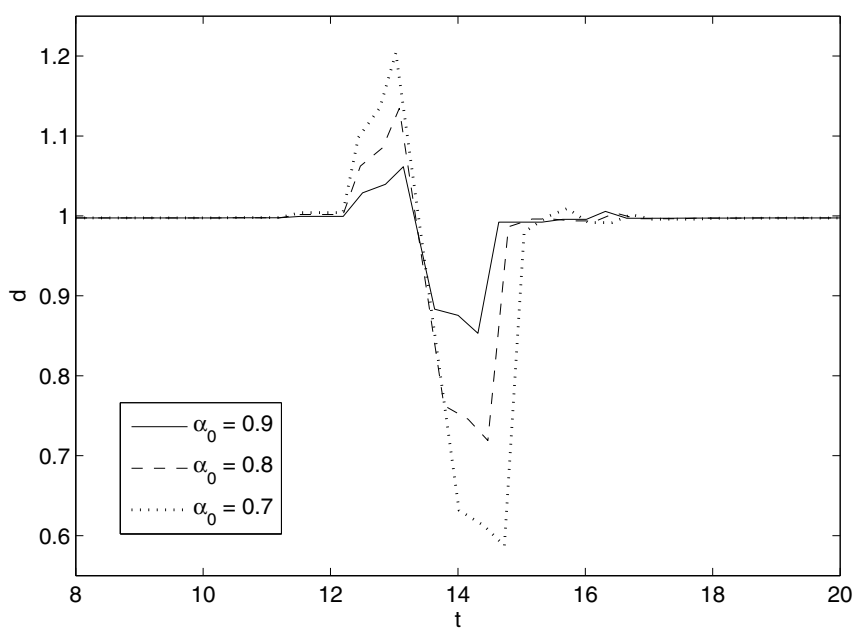

FIG. 5. Wave speed as a function of time for $\alpha=1$ and three values of $\alpha_{0}$, with $a=0.8116$ which gives $c \approx 1$.

to wave speeds near $c=1$ is almost instantaneous, once the interface has passed the defect.

Given wave speed estimates, we numerically compute the wave forms (4.12) with $\alpha_{0}=0.6$. Figure 6 has wave forms at $\xi^{*}=-0.6029$, where the front experiences speedup requiring a single variable wave speed $d_{-1}=1.1392$, and wave forms at $\xi^{*}=0.4794$, where the speed has significantly decreased, so $d_{0}=0.5552$. Each figure shows the effects of the defect on the front, the wave forms $\phi\left(\xi ; \xi^{*}\right)$ and the numerical solution of the evolution equation, and the difference between these wave forms. Since the solutions of the evolution equation are approximated with a maximum error estimate of $10^{-10}$, and the quadrature errors are better than $10^{-3}$, the errors shown in the lower plots are mostly due to errors in wave speeds, both computational errors and errors resulting from using the minimum requirement $\mathcal{R}=\{0\}$ and $\mathcal{S}=\{-1,0,1\}$. Nevertheless, the results do ensure that both the wave forms and the wave speeds are faithful to the true values. In addition, the upper plots show that $\chi\left(\xi^{*} ; \xi^{*}\right) \approx 0$ (up to the accuracy demonstrated in lower plots), giving numerical verification that the conditions (4.11) are satisfied.

5. Conclusion. In this paper we have considered front solutions to a bistable differential-difference equation with inhomogeneous discrete diffusion using a piecewise linear (McKean) reaction term. Our interest is in both stationary front and traveling front solutions. Due to the lack of translation invariance, the solutions we have obtained at a given time are not necessarily translations of the solutions at different times. However, by employing a traveling front-like formalism, we were able to determine details of the propagation, or lack of propagation, for solutions that behave much like traveling front solutions of problems with homogeneous diffusion.

We derived equilibrium solutions and the range of propagation failure for discrete media with a single defect. For media with multiple defects we provided a general formula for equilibrium solutions and explicit formulae that determine the range of propagation failure. We have shown that if a front solution is to cease propagating 

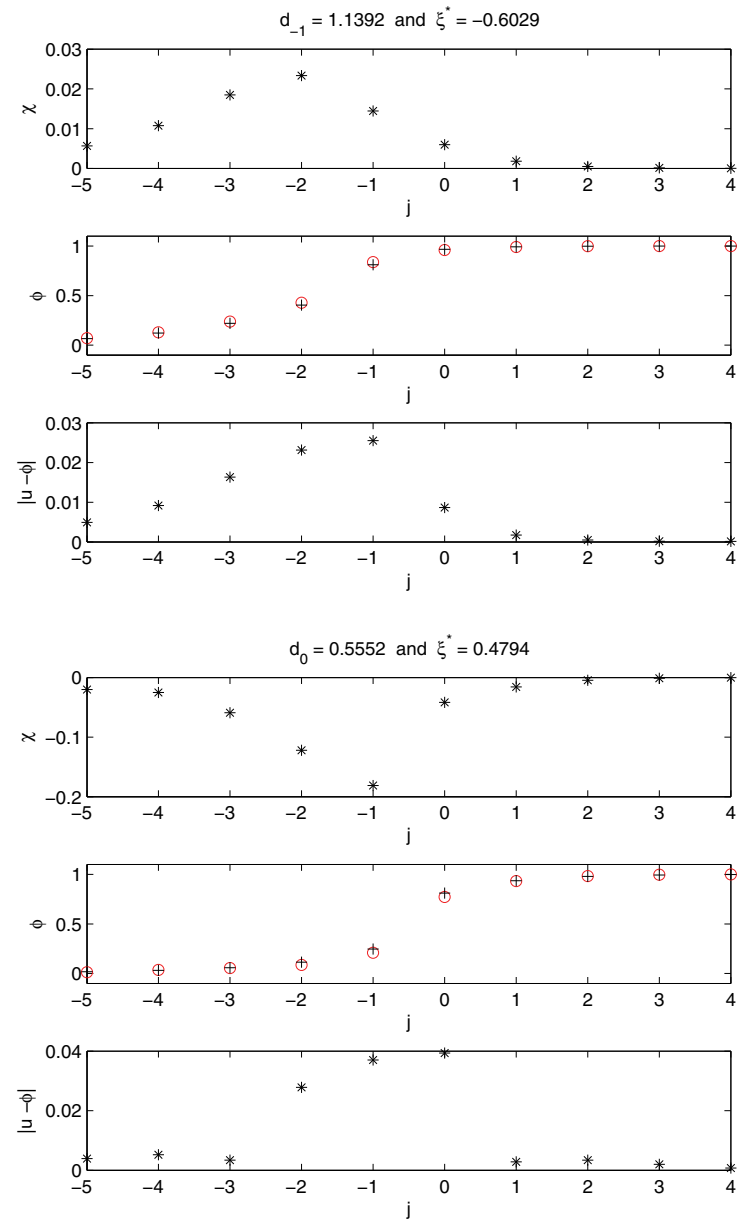

FIG. 6. For $\alpha=1, \alpha_{0}=0.6$, and $c=1$, wave forms at two positions, $\xi^{*}=-0.6029$ and $\xi^{*}=0.4794$, near the defect. These respective positions require variable wave speeds $d_{-1}$ and $d_{0}$, and for each position the following three plots are given. Top: $\chi=\varphi-\psi$, showing the difference between the solution with one defect and the solution with no defects. Middle: Wave forms represented by the numerical solution $u$ of the evolution equation (crosses) and the computed solution $\varphi$ obtained by Fourier transform (circles). Bottom: The difference $|u-\varphi|$.

due to a decrease in the diffusion coefficient at a single point, then this failure to propagate can occur only when the front is in a specific region near the defect. For multiple defects of the same size, the width of the interval of propagation failure is affected little by the number of defects, but the precise location of the pinned front largely depends on the number of defects. We have also found that it is not the size of the defect(s) that is most significant for determining the range of propagation failure, but rather the ratio to the constant diffusion coefficients that matters most.

Traveling front-like solutions are derived using a Fourier transform, and the result is a perturbation of the solutions that have been derived for the case of homogeneous diffusion. Essentially, the speed of the front at each lattice point must be allowed to change with time, and we approximate the solutions by allowing only a few of these wave speeds to vary with time, those closest to the defect region. To investigate the 
dynamics near the defect, we use high order approximations for the evolution equation to determine how a single wave speed varies with time as the front passes through the defect, and the results are verified by substitution into our derived solutions. Most interestingly, we find that the fronts experience speed-up immediately prior to the deceleration that results from approaching the defect, and once the front has passed the defect, it immediately returns to its former state.

\section{REFERENCES}

[1] D. G. Aronson, N. V. Mantzaris, and H. G. Othmer, Wave propagation and blocking in inhomogeneous media, Discrete Contin. Dynam. Systems, 13 (2005), pp. 843-876.

[2] P. W. Bates, X. Chen, And A. J. J. ChmaJ, Traveling waves of bistable dynamics on a lattice, SIAM J. Math. Anal., 35 (2003), pp. 520-546.

[3] J. Bell AND C. Cosner, Threshold behavior and propagation for nonlinear differentialdifference systems motivated by modeling myelinated axons, Quart. Appl. Math., 42 (1984), pp. $1-114$.

[4] J. W. CAHN, Theory of crystal growth and interface motion in crystalline materials, Acta Metallica, 6 (1960), pp. 554-561.

[5] J. W. Cahn, J. Mallet-Paret, and E. S. Van Vleck, Traveling wave solutions for systems of ODEs on a two-dimensional spatial lattice, SIAM J. Appl. Math., 59 (1998), pp. 455-493.

[6] A. Carpio and L. L. Bonilla, Depinning transitions in discrete reaction-diffusion equations, SIAM J. Appl. Math., 63 (2003), pp. 1056-1082.

[7] X. Chen, J. S. Guo, ANd C. C. Wu, Traveling waves in discrete periodic media for bistable dynamics, Arch. Ration. Mech. Anal., 189 (2008), pp. 189-236.

[8] S. N. Chow, J. Mallet-Paret, And W. Shen, Traveling waves in lattice dynamical systems, J. Differential Equations, 149 (1998), pp. 248-291.

[9] S. Coombes, G. J. Lord, And M. R. Owen, Waves and bumps in neuronal networks with axo-dendritic synaptic interactions, Phys. D, 178 (2003), pp. 219-241.

[10] C. E. Elmer, Finding stationary fronts for a discrete Nagumo and wave equation: Construction, Phys. D, 218 (2006), pp. 11-23.

[11] C. E. Elmer, The stability of stationary fronts for a discrete nerve axon model, Math. Biosci. Eng., 4 (2007), pp. 113-129.

[12] C. E. Elmer and E. S. VAN Vleck, Traveling wave solutions for bistable differential-difference equations with periodic diffusion, SIAM J. Appl. Math., 61 (2001), pp. 1648-1679.

[13] C. E. Elmer and E. S. Van Vleck, Spatially discrete FitzHugh-Nagumo equations, SIAM J. Appl. Math., 65 (2005), pp. 1153-1174.

[14] G. FATH, Propagation failure of traveling waves in discrete bistable medium, Phys. D, 116 (1998), pp. 176-190.

[15] J. A. FEROE, Existence and stability of multiple impulse solutions of a nerve equation, SIAM J. Appl. Math., 42 (1982), pp. 235-246.

[16] P. C. Fife And L. A. Peletier, Clines induced by variable selection and migration, Proc. R. Soc. London Ser. B, 214 (1981), pp. 99-123.

[17] A. ISERLES, On the numerical quadrature of highly oscillating integrals I: Fourier transforms, IMA J. Numer. Anal., 24 (2004), pp. 365-391.

[18] A. Iserles AND S. P. Norsett, On quadrature methods for highly oscillatory integrals and their implementation, BIT, 44 (2004), pp. 755-772.

[19] J. P. KeEner, Propagation and its failure in coupled systems of discrete excitable cells, SIAM J. Appl. Math., 47 (1987), pp. 556-572.

[20] J. P. KeEner, Propagation of waves in an excitable medium with discrete release sites, SIAM J. Appl. Math., 61 (2000), pp. 317-334.

[21] J. P. KeEner, Homogenization and propagation in the bistable equation, Phys. D, 136 (2000), pp. $1-17$.

[22] T. J. Lewis AND J. P. KeEner, Wave-block in excitable media due to regions of depressed excitability, SIAM J. Appl. Math., 61 (2000), pp. 293-316.

[23] J. Mallet-PARET, The Fredholm alternative for functional differential equations of mixed type, J. Dynam. Differential Equations, 11 (1999), pp. 1-48.

[24] J. Mallet-PARET, The global structure of traveling waves in spatially discrete dynamical systems, J. Dynam. Differential Equations, 11 (1999), pp. 49-128.

[25] H. McKean, Nagumo's equation, Adv. Math., 4 (1970), pp. 209-223.

[26] J. P. PAuwelussen, Nerve impulse propagation in the branching nerve system: A simple model, Phys. D, 4 (1981), pp. 67-88. 
[27] J. Rinzel And J. B. Keller, Traveling wave solutions of a nerve conduction equation, Biophys. J., 13 (1973), pp. 1313-1337.

[28] A. Scheel and E. S. VAn Vleck, Lattice differential equations embedded into reactiondiffusion systems, Proc. Roy. Soc. Edinburgh, 139 (2009), pp. 193-207.

[29] W. Shen, Traveling waves in time almost periodic structures governed by bistable nonlinearities I: Stability and uniqueness, J. Differential Equations, 159 (1999), pp. 1-54.

[30] W. Shen, Traveling waves in time almost periodic structures governed by bistable nonlinearities II. Existence, J. Differential Equations, 159 (1999), pp. 55-101.

[31] J. Sneyd And J. Sherratt, On the propagation of calcium waves in an inhomogeneous media, SIAM J. Appl. Math., 57 (1997), pp. 73-94.

[32] G. Teschl, Jacobi Operators and Completely Integrable Nonlinear Lattices, Math. Surveys Monogr. 72, American Mathematical Society, Providence, RI, 2000.

[33] L. Truskinovsky And A. Vainchtein, Kinetics of martensitic phase transitions: Lattice model, SIAM J. Appl. Math., 66 (2005), pp. 533-553.

[34] W.-P. WANG, Multiple impulse solutions to McKean's caricature of the nerve equation. IExistence, Comm. Pure Appl. Math., 41 (1988), pp. 71-103.

[35] W.-P. WANG, Multiple impulse solutions to McKean's caricature of the nerve equation. II. Stability, Comm. Pure Appl. Math., 41 (1988), pp. 997-1025.

[36] J. Yang, S. Kalliadasis, J. H. Merkin, and S. K. Scott, Wave propagation in spatially distributed excitable media, SIAM J. Appl. Math., 63 (2002), pp. 485-509.

[37] B. ZINnER, Stability of traveling wavefronts for the discrete Nagumo equation, SIAM J. Math. Anal., 22 (1991), pp. 1016-1020.

[38] B. ZINNER, Existence of traveling wavefront solutions for the discrete Nagumo equation, J. Differential Equations, 96 (1992), pp. 1-27.

Copyright (c) by SIAM. Unauthorized reproduction of this article is prohibited. 